\title{
Tonic engagement of nicotinic receptors bidirectionally controls striatal spiny projection neuron spike timing
}

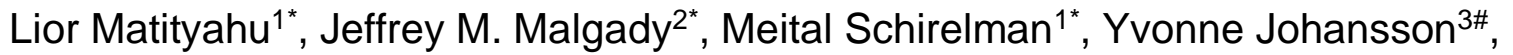 \\ Jennifer Wilking ${ }^{2}$, Gilad Silberberg ${ }^{3}$, Joshua A. Goldberg ${ }^{1 \dagger}$ and Joshua L. Plotkin ${ }^{2 \dagger}$
}

${ }^{1}$ Department of Medical Neurobiology, Institute of Medical Research Israel-Canada, The Faculty of Medicine, The Hebrew University of Jerusalem, 9112102, Jerusalem, Israel 2Department of Neurobiology and Behavior, Stony Brook University School of Medicine, Stony Brook University, NY, 11794, USA

${ }^{3}$ Department of Neuroscience, Karolinska Institutet, 17177 Stockholm, Sweden \#Current Address: Sainsbury Wellcome Centre for Neural Circuits and Behaviour, University College London, London, UK

${ }^{*}$ Authors contributed equally to this work

†Equal corresponding authors 


\begin{abstract}
Striatal spiny projection neurons (SPNs) transform convergent excitatory corticostriatal inputs into an inhibitory signal that shapes basal ganglia output. This process is finetuned by striatal GABAergic interneurons (GINs), which receive overlapping cortical inputs and mediate rapid corticostriatal feedforward inhibition of SPNs. Adding another level of control, cholinergic interneurons (CINs), which are also vigorously activated by corticostriatal excitation, can 1) disynaptically inhibit SPNs by activating $\alpha 4 \beta 2$ nicotinic acetylcholine receptors ( $\mathrm{nAChRs}$ ) on various GINs and 2) directly modulate corticostriatal synaptic strength via pre-synaptic $\alpha 7 \mathrm{nAChR}$ receptors. Measurements of the disynaptic inhibitory pathway, however, indicate that it is too slow to compete with direct GIN-mediated feed-forward inhibition. Moreover, functional nAChRs are also present on populations of GINs that do not respond to phasic activation of CINs, such as parvalbumin-positive fast-spiking interneurons (PV-FSIs), making the overall role of $\mathrm{nAChRs}$ in shaping striatal synaptic integration unclear. Using acute striatal slices we show that upon synchronous optogenetic activation of corticostriatal projections, blockade of $\alpha 7 \mathrm{nAChRs}$ delayed SPN spikes, whereas blockade of $\alpha 4 \beta 2 \mathrm{nAChRs}$ advanced SPN spikes and increased postsynaptic depolarizations. The nAChRdependent inhibition was mediated by downstream GABA release, and data suggest that the GABA source was not limited to GINs that respond to phasic CIN activation. In particular, the observed spike-advancement caused by nAChR blockade was associated with a diminished frequency of spontaneous inhibitory postsynaptic currents in SPNs, and a parallel hyperpolarization of PV-FSIs. Taken together, we describe opposing roles for tonic (as opposed to phasic) engagement of $n A C h R s$ in striatal function. We conclude that tonic activation of nAChRs by CINs both sharpens the temporal fidelity of corticostriatal signaling via pre-synaptic $\alpha 7 \mathrm{nAChRs}$ and maintains a GABAergic brake on cortically-driven striatal output, processes that may shape SPN spike timing, striatal processing and synaptic plasticity.
\end{abstract}

Key Words: methyllycaconitine; mecamylamine; DHßE; channelrhodopsin-2; striatal microcircuitry; tonically active neurons; paired-pulse ratio; postsynaptic potential; afterdepolarization 


\section{Introduction}

As the main input nucleus of the basal ganglia, the striatum receives convergent cortical synaptic information, generating a computationally transformed interpretation of this information and relaying it to intermediate and output nuclei of the basal ganglia. Striatal output is carried exclusively by GABAergic striatal spiny projection neurons (SPNs), which account for $\sim 95 \%$ of all striatal neurons (Gerfen and Surmeier 2011, Silberberg and Bolam 2015). The remaining $5 \%$ of striatal neurons are interneurons, most of which are GABAergic interneurons (GINs). There are several molecularly distinct classes of GINs that exhibit diverse intrinsic firing patterns and patterns of intrastriatal connectivity (Tepper, Tecuapetla et al. 2010, Muñoz-Manchado, Bengtsson Gonzales et al. 2018, Assous and Tepper 2019). In the context of feedforward cortico- and thalamostriatal transmission the GINs' main function lies in the feedforward inhibition they provide to SPNs (Tepper, Koós et al. 2004, Assous, Kaminer et al. 2017). Such inhibition can fashion striatal output by perturbing the precise timing of SPNs or prevent their spiking altogether. The only non-GABAergic interneurons are the large, aspiny, cholinergic interneurons (CINs) (DiFiglia 1987, Tepper and Bolam 2004, Plotkin and Goldberg 2019, Poppi et al. 2021). Despite their small numbers (approximately $1 \%$ of striatal neurons) the acetylcholine (ACh) they release influences the entire striatal microcircuitry. A single CIN axon can cover a third of the striatum (in linear dimension) and has ACh release sites every few micrometers (DiFiglia 1987). In fact, the striatum has the highest expression of cholinergic markers in the entire CNS (Mesulam, Mash et al. 1992, Contant, Umbriaco et al. 1996). CINs are autonomous pacemakers (Bennett and Wilson 1999) that are largely identified as the tonically active neurons (TANs) of the striatum

(Kimura, Rajkowski et al. 1984, Wilson, Xu et al. 1990, Aosaki, Tsubokawa et al. 1994, Aosaki, Kimura et al. 1995, Morris, Arkadir et al. 2004), firing 3-10 spikes/s in vivo. On the backdrop of this ongoing discharge, the main signal they convey in vivo is a pause in response to primary reward or salient stimuli associated with reward (Aosaki, Tsubokawa et al. 1994, Morris, Arkadir et al. 2004, Goldberg and Reynolds 2011, Apicella 2017). The duration of the pause in response to a brief conditioned sensory stimulus is on the order of 200-300 ms (Kimura, Rajkowski et al. 1984, Raz, Feingold et al. 1996, Apicella, Legallet et al. 1997). 
CINs regulate SPNs in 3 main ways. The best characterized regulation is exerted via muscarinic ACh receptors (mAChRs). Activation of presynaptic and postsynaptic mAChRs on SPNs modulates synaptic transmission, synaptic plasticity and the intrinsic excitability of SPNs by modulating various voltage-activated $\mathrm{Ca}^{2+}$ and $\mathrm{K}^{+}$channels (Akins, Surmeier et al. 1990, Calabresi, Centonze et al. 1999, Gabel and Nisenbaum 1999, Day, Wokosin et al. 2008, Goldberg, Ding et al. 2012, Zucca, Zucca et al. 2018). CINs influence SPNs via nicotinic ACh receptors (nAChRs) as well, albeit indirectly. Activation of $\mathrm{nAChRs}$ on striatal dopaminergic fibers can evoke dopamine (DA) release (Zhou, Liang et al. 2001, Cachope, Mateo et al. 2012, Threlfell, Lalic et al. 2012) which, in turn, can modulate the intrinsic excitability of SPNs and synaptic transmission and plasticity at synaptic inputs. All of the above influences are mediated by GPCR-linked ACh and DA receptors, which means that they are unlikely to dynamically affect the moment-by-moment processing and transmission of excitatory inputs to SPNs. Indeed, a recent study in which CINs were silenced optogenetically in vivo has put a lower bound on how fast mAChR-mediated effects can come into play. When CINs are synchronously silenced for over $500 \mathrm{~ms}$, SPNs begin to show signs of mAChRdependent reductions in excitability. However, that effect comes into play only after a 400 ms delay (Zucca, Zucca et al. 2018). The only known mechanisms by which CINs can rapidly affect SPN activity (sooner than $400 \mathrm{~ms}$ ) involve nicotinic or muscarinic modulation of synaptic glutamate release or a4 $32 \mathrm{nAChR}$-dependent GABA release onto the SPNs' ionotropic GABAA receptors (Pakhotin and Bracci 2007, English, lbanezSandoval et al. 2011, Faust, Assous et al. 2015, Faust, Assous et al. 2016, Assous, Kaminer et al. 2017, Assous 2021). The $\alpha 4 \beta 2$ nAChRs that drive disynaptic GABA release are located on GINs (English, Ibanez-Sandoval et al. 2011, Faust, Assous et al. 2015), and on dopaminergic nigrostriatal terminals (Nelson, Hammack et al. 2014).

Because CINs receive massive excitatory cortical and thalamic input (Lapper and Bolam 1992, Thomas, Smith et al. 2000) it is conceivable that their disynaptic inhibition of SPNs comes into play during bouts of cortical or thalamic activity. However, this raises a conceptual problem. Because cortex and thalamus can activate feedforward 
inhibition to SPNs via various striatal GINs (e.g., cortex $\rightarrow$ GIN $\rightarrow$ SPN), what is gained by recruiting an additional pathway that is necessarily slower and less reliable due to its additional synapse (e.g., cortex $\rightarrow \mathrm{CIN} \rightarrow \mathrm{GIN} \rightarrow$ SPN)? Moreover, what is the role of nAChRs on GINs, such as the parvalbumin-positive fast-spiking interneurons (PV-FSIs) that do not respond to direct activation of CINs (English, Ibanez-Sandoval et al. 2011, Nelson, Hammack et al. 2014)? This conceptual problem exists only if we assume that the role of CINs in modulating SPN function through the activation of nAChRs is anchored around their phasic activation by excitatory afferents. In this study we demonstrate that ongoing CIN activity exerts opposing influences on SPNs via tonic engagement of nAChRs in different pathways: tonic activation of pre-synaptic $\alpha 7$ nAChRs sharpens the temporal fidelity of responses to cortical inputs, while tonically activated $\alpha 4 \beta 2$ nAChRs on GINs provide a constant brake on SPN excitation. We uncover these nicotinic effects using transgenic mice that express channelrhodopsin-2 (ChR2) in corticostriatal fibers (Arenkiel, Peca et al. 2007). Activation of these fibers creates a competition between monosynaptic cortical excitation and polysynaptic feedforward inhibition to SPNs. We show that nAChRs on distinct pre- and postsynaptic pathways are capable of bidirectionally controlling SPN spike timing with millisecond precision.

\section{Results}

To interrogate microcircuit-level responses of SPNs to cortical excitation of the striatum, we generated acute ex vivo brain slices from Thy1-ChR2 mice, which nominally express ChR2 in cortical neurons (Arenkiel, Peca et al. 2007, Aceves Buendia, Tiroshi et al. 2019). Full-field optogenetic stimulation with a $470 \mathrm{~nm}$ LED engaged corticostriatal afferents and generated in SPNs either: excitatory postsynaptic potentials (EPSPS) for low LED intensities, or - in the suprathreshold condition - an action potential (AP) followed by an after depolarization (ADP) lasting 10s of milliseconds (Fig. 1a). For the remainder of this study we tuned the LED intensity to generate a just-suprathreshold response so that AP latency and ADP amplitude could be compared among SPNs. Blockade of nAChRs with mecamylamine (mec; $10 \mu \mathrm{M}$ ) enhanced the engagement of SPN responses (Fig. 1b), as it shortened AP latency (Fig. 1c) and increased both the 
ADP (Fig. 1d) and subthreshold EPSP amplitudes (Fig. 1e). These effects were replicated in the presence of the $\alpha 4 \beta 2 \mathrm{nAChR}$-selective antagonist, $\mathrm{DH} \beta E(10 \mu \mathrm{M}, \mathrm{AP}$ latency: $\mathrm{n}=8$ SPNs, $\mathrm{p}=7.8 \cdot 10^{-3}$, SRT; ADP amplitude: $\mathrm{n}=8$ SPNs, $\mathrm{p}=0.039$, SRT; data not shown). Because the resting membrane potential of SPNs is very hyperpolarized compared to the depolarized potential from which spikes typically occur in vivo [the so-called "up state" (Wilson and Groves 1981, Stern, Jaeger et al. 1998)], we repeated the experiment while holding the SPN with a constant positive current injection in the just-subthreshold region (e.g., in the $-55 \mathrm{mV}$ to $-50 \mathrm{mV}$ range). Mecamylamine shortened the latency to AP during this somatically-mimicked "up state" as well (Fig. 1f-g).

Since SPNs themselves do not express nAChRs, the action of mecamylamine must be indirect. Functional $\mathrm{nAChR}$ are expressed at multiple loci of the striatal circuit, including afferent axonal terminals and intrastriatal GABAergic interneurons (Nelson, Hammack et al. 2014, Faust, Assous et al. 2016, Abudukeyoumu, Hernandez-Flores et al. 2019, Assous 2021). While the broad antagonistic actions of mecamylamine and DH $\beta E$ should block nicotinic receptors at most of these loci, $\alpha 7 \mathrm{nAChRs,} \mathrm{which} \mathrm{are} \mathrm{predominantly}$ expressed at corticostriatal axon terminals, are likely spared (Solinas, Scherma et al. 2007, Licheri, Lagström et al. 2018, Assous 2021). Indeed, mecamylamine did not have a significant effect on the strength or release probability of corticostriatal glutamatergic afferents, as measured by local electrical stimulation (Fig. 2a-c).

The lack of an effect of mecamylamine on presynaptic release probability does not exclude a direct influence of tonic activation of $\alpha 7 \mathrm{nAChRs}$ on SPN spike timing. Indeed, blockade of $\alpha 7 \mathrm{nAChRs}$ with (2-5 $\mu \mathrm{M})$ MLA actually delayed AP latency (Fig. 2d,e). Thus, tonic engagement of nAChRs has opposing modulatory effects on SPN spike timing. If presynaptic corticostriatal nAChRs can advance an SPN spike, how then does mecamylamine, which has no presynaptic action, shorten SPN AP latency as well? Cholinergic interneurons form a disynaptic circuit with SPNs, which is mediated by neuropeptide Y-neurogliaform (NPY-NGF) GINs and perhaps other classes of nAChRexpressing GABAergic interneurons (GINs) in a nAChR-dependent manner (English, 
Ibanez-Sandoval et al. 2011, Elghaba, Vautrelle et al. 2016, Faust, Assous et al. 2016, Assous, Kaminer et al. 2017, Tepper, Koós et al. 2018). Consistent with the involvement of such a polysynaptic circuit, blockade of GABAergic transmission with the GABA and $\mathrm{GABA}_{\mathrm{B}}$ receptor antagonists SR-95531 (10 $\left.\mu \mathrm{M}\right)$ and CGP-55845 (2 $\left.\mu \mathrm{M}\right)$ respectively, mimicked and the effect of mecamylamine on spike timing and ADP amplitude (Fig. 3ac). Blockade of GABA receptors fully occluded mecamylamine's effect on both spike latency and ADP amplitude (Fig. 3a-c), further implicating a CIN-GIN-SPN disynaptic circuit in mediating the actions of $n A C h R s$. Strikingly, mecamylamine prevented GABA receptor antagonists from further advancing spike timing or enhancing ADP amplitude, suggesting that $n A C h R$ engagement can saturate GABAergic inhibition (Fig. 3d-f).

CINs receive converging excitatory inputs from the thalamus as well as the cortex (Lapper and Bolam 1992, Thomas, Smith et al. 2000). Indeed, targeted recordings from CINs confirmed that they are robustly engaged by our corticostriatal stimulation protocol, even displaying a lower stimulation threshold and shorter average delay to spike than SPNs (Fig. 4a-c). Despite the observed high fidelity and speed of corticallyevoked CIN spiking, a comparison of the temporal dynamics of synchronous cortical engagement of SPN inhibition (latency of approximately $5 \mathrm{~ms}$, Fig. 5a) vs synchronous CIN engagement of GINs [latency of approximately $11 \mathrm{~ms}$, (English, Ibanez-Sandoval et al. 2011, Nelson, Hammack et al. 2014)] shows that phasic activation of CINs is still not sufficiently fast to account for the mecamylamine-induced spike delay we observed in SPNs (Fig. 5b). In particular, even if there are sufficient numbers of CINs that respond instantaneously to cortical activation due to their ongoing activity, the earliest latency at which their phasic inhibition can influence SPNs is 11 ms later (English, IbanezSandoval et al. 2011, Nelson, Hammack et al. 2014). However, SPN spikes are being delayed beginning $6 \mathrm{~ms}$ after stimulation (Fig. 5b magenta), which cannot be explained by phasic CIN activation.

If phasic engagement of $\mathrm{nAChRs}$ by synaptically evoked CIN activity cannot pragmatically account for the observed delay in SPN spike initiation, how does blocking nAChRs advance synaptically evoked SPN spiking? Given the autonomous 
pacemaking nature of CINs, perhaps the explanation is that tonic, rather than phasic, activation of $\mathrm{nAChRs}$ is key to the phenomenon. There are three obvious mechanisms that could be at play in this scenario: 1) ongoing $n A C h R$ activation (either tonic or phasic activation that is not time-locked with the corticostriatal stimulation event leading to SPN spiking) may decrease SPN intrinsic excitability indirectly, likely by altering ongoing neuromodulator release (Zhou, Wilson et al. 2002, Rice and Cragg 2004) 2) the effect on SPN spiking may be a non-specific drug effect of mecamylamine (this is unlikely, since DHßE had the same effects) or 3) tonic engagement of somatodendritic $\mathrm{nAChRs}$ may produce an ongoing depolarization of GINs, ultimately priming or accentuating corticostriatal feedforward inhibition.

To test the possibility that blockade of ongoing $\mathrm{nAChR}$ activation increases SPN excitability, we measured the current-voltage relationship of SPNs before and during mecamylamine application. While there is no indication that our effect of mecamylamine on spike timing or ADP amplitude is bimodal and specific to direct pathway SPNs (dSPNs) or indirect pathway SPNs (iSPNs), dSPNs and iSPNs display differences in basal excitability (Gertler, Chan et al. 2008), which can complicate interpretation. We therefore performed these experiments in transgenic mice where dSPNs and iSPNs could be identified by their fluorescent label (Shuen, Chen et al. 2008, Ade, Wan et al. 2011) (Fig. 6a). Mecamylamine had no significant effect on the resting membrane potential or rheobase current of either SPN type (Fig. 6b,c). Furthermore, mecamylamine did not significantly alter the time to spike in response to somatic rheobase current injection or the latency to first spike in an AP train induced by suprathreshold somatic current injection (data not shown). Given that most data to this point came from pooled populations of SPNs, and that we observed no SPN-type specific effects of mecamylamine on SPN excitability, we performed additional analysis on pooled SPN data. While rheobase currents were unaffected, mecamylamine decreased the responsiveness to several sub-rheobase amplitude currents, arguing that if anything mecamylamine may decrease the excitability of SPNs in some regards (Fig. 6d). Mecamylamine had no effect on the firing rate of SPNs (Fig. 6e), nor did it alter IV properties of unidentified SPNs recorded from Thy1-ChR2 mice ( $n=55$ SPNs in control, 
$\mathrm{n}=9$ SPNs in mecamylamine, data not shown).Taken together, spike advancement induced by nAChR blockade cannot be explained by changes in SPN intrinsic excitability.

Since mecamylamine did not increase the intrinsic excitability of SPNs, we tested if nAChR blockade attenuated the basal inhibitory GABAergic influence that they are under. Indeed, mecamylamine significantly reduced the frequency of spontaneous inhibitory postsynaptic currents (sIPSCs) in a mixed population of SPNs (Fig. 7a-c). As SPNs are quiescent in acute slices and lack nAChRs, the source of these attenuated sIPSCs is likely GINs. Indeed, various classes of GINs are excited, and their spontaneous activity is elevated, by tonic activation of nAChRs (Luo, Janssen et al. 2013, Muñoz-Manchado, Foldi et al. 2014, Ibáñez-Sandoval, Xenias et al. 2015, Elghaba, Vautrelle et al. 2016, Tepper, Koós et al. 2018). Because PV-FSIs convey strong feedforward inhibition to SPNs (Koós and Tepper 1999, Tepper, Koós et al. 2004, Planert, Szydlowski et al. 2010), but do not respond to phasic CIN (English, Ibanez-Sandoval et al. 2011), we made targeted current clamp recordings from PV-Cre $x$ Ai9-tdTomato transgenic mice (Johansson and Silberberg 2020) and tested the effect of $n A C h R$ blockade on their excitability (Fig. 7d). Indeed, blocking nAChRs by bath application of DHßE significantly hyperpolarized the resting membrane potential of PVFSIs (Fig. 7e) and augmented AP kinetics (Fig. 7f-g). Thus, tonic activation of nAChRs depolarizes the resting membrane potential of PV-FSIs and other GINs (Elghaba 2016), continuously holding them closer to the AP threshold, and thereby priming them to transmit cortically-driven feedforward inhibition more efficiently to SPNs. Interestingly, the dependence upon trailing "primed" GIN input may explain why mecamylamine was capable of diminishing the amplitude of relatively slow rising EPSPs (Fig. 1e) but not faster peaking EPSCs (Fig. 2b). In summary, the tonic activity of CINs recruits either: i) presynaptic nAChRs to increase the fidelity of SPN responses to cortical inputs, or ii) specific GINs to provide a "nicotinic brake" on SPN responsiveness. Such mechanisms could allow SPNs to be more receptive of cortical inputs when CINs pause their firing in response to salient inputs or stimuli associated with reward. 


\section{Discussion}

The present study describes a novel role for tonic nAChR activation in shaping striatal output. Decades of research have firmly established CINs as important modulators mostly via muscarinic ACh receptors (Goldberg, Ding et al. 2012, Zucca, Zucca et al. 2018, Assous 2021) - of cortico- and thalamo- striatal synaptic integration and determinants of striatal output (Akins, Surmeier et al. 1990, English, Ibanez-Sandoval et al. 2011, Abudukeyoumu, Hernandez-Flores et al. 2019). Despite the fact that CINs are autonomous pacemakers, nearly all studies examining their influence on the precise temporal output of the striatum, particularly via nAChRs, have been centered around the consequences of their phasic engagement (English, Ibanez-Sandoval et al. 2011, Faust, Assous et al. 2016, Dorst, Tokarska et al. 2020). Here we report that ongoing, non-phasic activation of nAChRs accounts for a basal elevation in GABAergic tone, which dampens the response of SPNs to convergent cortical inputs and ultimately delays striatal output.

\section{Basal cholinergic regulation of striatal output- a postsynaptic "nicotinic brake"?}

CINs can robustly evoke GABAergic inhibition in the striatum by engaging somatodendritic nAChRs on specific classes of local GINs, in particular those expressing NPY (English, Ibanez-Sandoval et al. 2011, Luo, Janssen et al. 2013). This cell-type specific nAChR-dependent mechanism has been demonstrated using electrical or optogenetic stimulation to drive phasic, stimulation-locked inhibition. Somewhat puzzling, however, are reports that certain GINs, including PV-FSIs, cannot be phasically activated by CINs [or by cholinergic afferents from the midbrain (Dautan, Huerta-Ocampo et al. 2020)] even though they express the requisite somatodendritic nAChRs (Koós and Tepper 2002, English, Ibanez-Sandoval et al. 2011, Nelson, Hammack et al. 2014). Our finding that tonic engagement of nAChRs enhances the excitability of FSIs, as has been shown previously (Koós and Tepper 2002, Luo, Janssen et al. 2013), offers a physiological role for these receptors, and supports the notion that volume transmission of ACh takes place in the striatum (Picciotto, Higley et al. 2012). 
Tonic nAChR excitation of GINs helps clarify another puzzling observation. It is obvious why GABAR blockers occlude the effect of mecamylamine on AP latency and ADP amplitude in SPNs- the relevant GABARs are synaptically downstream of the nAChRs on GINs. But why is it that mecamylamine seems to "saturate" the effect of GABAR inhibition on SPN AP latency and ADP? In other words, why does blockade of GABARs have no additional effect on AP latency or ADP when applied after mecamylamine, when phasic CIN activation only engages a select few types of GINs? If nAChRs were recruited solely by phasic activation by CINs, then the addition of GABAR blockers after mecamylamine should further shorten the AP latency and increase ADP amplitude by attenuating the influence of other GINs (such as PV-FSIs) that are not phasically activated by nAChRs (English, Ibanez-Sandoval et al. 2011, Nelson, Hammack et al. 2014). A "priming" effect of tonic $n A C h R$ activation on GINs that are not directly driven to spike by CINs could explain the seemingly oversized influence of nicotinic signaling on GABAergic inhibition. For this reason, determining which GINs are responsible for the effect may be ill-posed. Instead, our strategy was to demonstrate that in the presence of $\mathrm{nAChR}$ antagonists, SPNs receive less tonic inhibitory input, and that one of the GINs, the FSI - that has been shown to dominate the feedforward cortical inhibition (Koós and Tepper 1999, Johansson and Silberberg 2020) - is more hyperpolarized at rest (Fig. 7E). While the effect on PV-FSI resting membrane potential that we observed was modest, it is likely that multiple GINs participate in this mechanism, including low-threshold spiking (LTS) and $\mathrm{TH}^{+}$GINs, that are autonomously active or exhibit long plateaus (Beatty, Sullivan et al. 2012, Ibáñez-Sandoval, Xenias et al. 2015) and therefore likely (like CINs) to respond rapidly to cortical inputs (Johansson and Silberberg 2020). Indeed, LTS neurons' spontaneous firing is slowed down considerably by mecamylamine, even though the effect on membrane voltage was on the order of what we observed in PV-FSIs (Elghaba, Vautrelle et al. 2016).Thus, various GINs are capable of laying the groundwork for a sort of 'nicotinic brake' present during basal circuit activity, attenuating and delaying striatal output. 
Under what conditions will the 'nicotinic brake' be lifted?

Through their continuous autonomous discharge, CINs tonically-release ACh in the striatum (Zhou, Wilson et al. 2002). While a prolonged exposure of striatal nAChRs to $\mathrm{ACh}$ is bound to desensitize them, it has been argued that the fast action of striatal acetylcholinesterase (AChE) prevents nAChR desensitization allowing for the maintenance of tonic nAChR activation in the striatum (Zhou, Wilson et al. 2002). So if tonic nAChR activation normally puts a brake on cortical feedforward inhibition, under what circumstances is this brake removed? The tonically active neurons (TANs) of the primate striatum, which are comprised mainly of CINs (Wilson, Xu et al. 1990, Aosaki, Kimura et al. 1995), acquire a synchronized pause response (Raz, Feingold et al. 1996) in their tonic firing that lasts several hundred milliseconds in response to primary reward or, through conditioning, to salient stimuli associated with reward (Kimura, Rajkowski et al. 1984, Apicella, Legallet et al. 1997, Goldberg and Reynolds 2011, Bradfield, BertranGonzalez et al. 2013). This pause duration should be sufficient - particularly while AChE rapidly clears the extracellular space from ACh - to deactivate the nAChRs, thereby removing the nicotinic brake.

Our study complements a recent study that described how CINs impact SPN neural dynamics in vivo (Zucca, Zucca et al. 2018). In that study it was shown that when CINs are optogenetically silenced in a synchronous-fashion for a sufficiently long period SPNs become hyperpolarized, less excitable and short-term corticostriatal plasticity is dampened. However, these effects commence only $400 \mathrm{~ms}$ after CINs are silenced. Until that point SPN excitability is unaffected. Thus, that study remained agnostic about the effect of CIN pauses that are shorter than 400 ms long on SPNs. Because physiological TAN pauses in response to sensory cues are typically over after $300 \mathrm{~ms}$ (Kimura, Rajkowski et al. 1984, Apicella, Legallet et al. 1997), the mAChR-mediated curbing of SPN excitability and responsiveness revealed in that and other studies (Ebihara, Yamamoto et al. 2013) may not be relevant to the role of the shorter, more physiological CIN pauses. Assuming that the nAChR-dependent mechanism we revealed can come into play sooner, our findings suggest that there are two dichotomous phases to the effect of synchronous CIN pauses: an 'early' period that is 
dominated by the consequences of removing tonic $\mathrm{nAChR}$ engagement, and a more delayed period driven by diminished activation of $\mathrm{mAChRs}$, where the responsiveness of SPNs to incoming cortical input is weakened, as shown previously (Zucca, Zucca et al. 2018).

\section{Implications for tuning striatal circuit activity}

Our data suggest that as CIN pauses develop several things will happen: tonic nAChRdriven boosting of corticostriatal synaptic inputs will be lost, but ongoing GABAergic inhibition will be relieved. This could have the effect of raising the threshold for spike generation, but sharpening the timing of spiking by several milliseconds once that threshold is reached, ultimately shaping the moment-by-moment processing of striatal output. Because the TAN pause usually coincides with the arrival of a salient stimulus, it is precisely a moment when SPNs need to be more attuned to cortical input and respond more reliably. Given the relatively higher affinity of $\alpha 4 \beta 2$ vs $\alpha 7$ nAChRs (Albuquerque, Pereira et al. 2009), it is feasible that the initial response to a salient stimulus (and drop in ACh levels) would be a rapid overall reduction in the responsivity of SPNs to cortical inputs, followed by a temporal sharpening of any proceeding spikes that are induced. Moreover, seminal experiments in primates have shown that CIN pauses become more prominently and reliably evoked by sensory stimuli over the course of Pavlovian conditioning (Aosaki, Tsubokawa et al. 1994, Aosaki, Kimura et al. 1995). Together, this implies that removal of the 'nicotinic brake' may be a crucial component of striatal learning. While a several milliseconds advancement in spike timing may not seem like much in the context of 100s of milliseconds long CIN pauses or SPN state transitions, it may be ample time to move a spike into or out of the time window for NMDA receptor-dependent coincidence detection to occur (Hao and Oertner 2012).

Both dSPNs and iSPNs robustly support spike timing dependent plasticity (STDP) (Pawlak and Kerr 2008, Shen, Flajolet et al. 2008). Under permissive conditions, constraining the relative timing of SPN APs to occur 5 milliseconds after a corticostriatal synaptic burst efficiently potentiates the active synapses (Shen, Flajolet et al. 2008). In 
this view, spike advancement on the time scale we observe may be sufficient to bring the spike temporally close enough to NMDA receptor activation to engage the signaling cascades that are crucial for long term potentiation. Such subtle nAChR-dependent tuning could be a way of 'distancing' those same cortical afferents from SPN activity under conditions of basal activity, allowing for the synapses encoding a particular sensorimotor cue to become potentiated only when deemed appropriate by a learned cholinergic signal.

The extent to which tonic engagement of $\mathrm{nAChRs}$ will be capable of influencing GABA release and SPN responses to cortical inputs will likely depend on the capacity of AChE activity to both allow for the detection of a CIN pause, and minimize nAChR desensitization (Zhou, Wilson et al. 2002). This brings up an important corollary of the 'nicotinic brake' hypothesis: the putative mechanism by which CIN pauses remove the nicotinic brake may be most robust in the AChE-enriched compartment of the striatum, also known as the matrix (as opposed to striosomes) (Graybiel and Ragsdale 1978, Brimblecombe and Cragg 2017, Prager and Plotkin 2019). Since the matrix constitutes $\sim 85 \%$ of the striatum, our data likely reflect the situation in that compartment (Prager and Plotkin 2019). While coordinated CIN activation can interrupt the timing of ongoing APs in both matrix and striosome SPNs (Crittenden, Lacey et al. 2017), the degree of tonic nAChR engagement and existence of a 'nicotinic brake' in striosomes is unclear.

Taken together, our data suggest an additional role for cholinergic signaling in shaping striatal activity. While a great deal is known about how ACh can impact SPN function through metabotropic muscarinic receptors, nearly all studies of the 'faster' nAChR arm of ACh signaling have focused on its phasic activation. The data in this study demonstrate that $\mathrm{nAChR}$ signaling is not constrained to times of phasic CIN engagement, and tonic activation of nAChRs places a 'brake' on SPN activity that may help guide the fine tuning of striatal output. 


\section{Materials and Methods}

Animals

All experimental procedures on mice adhered to and received prior written approval from the Institutional Animal Care and Use Committees of the Hebrew University of Jerusalem and of Stony Brook University and of the local ethics committee of Stockholm, Stockholms Norra djurförsöksetiska nämnd. Experiments were conducted on various transgenic mice. For optogenetic activation of corticostriatal fibers, we used 1-4 months old male and female homozygous transgenic Thy1-ChR2 mice [B6.CgTg(Thy1-COP4/EYFP)18Gfng/J] that express channelrhodopsin-2 under the Thy1 promoter were used. Thy1-ChR2 mice express ChR2 in cortical afferents under the thymus cell antigen 1 (Thy1) promoter. Thy 1 is generally expressed in the axons of layer V pyramidal neurons, limbic system, midbrain and brainstem (Arenkiel, Peca et al. 2007) . For experiments measuring paired pulse ratios, neuronal excitability and spontaneous GABAergic events, we used 1.5-4 month old male and female C57bl/6 mice crossed with one of two BAC transgenic lines (where indicated): drd1-tdTomato (labeling dSPNs) or drd2-eGFP (labeling iSPNs) (Shuen, Chen et al. 2008, Ade, Wan et al. 2011). For PV-FSI recordings, both male and female mice (p59-p65) were used in this study. Mice were group-housed under a $12 \mathrm{hr}$ light / dark schedule and given ad libidum access to food and water. The PV-Cre (stock \#017320, the Jackson laboratory) mouse line was crossed with a homozygous tdTomato reporter mouse line ('Ai9', stock \#007909, the Jackson laboratory) to allow identification of FSls based on the expression of a fluorescent marker protein. The Cre line was heterozygous and maintained on a wild-type C57BL/6J background (stock \# 000664, the Jackson Laboratory).

\section{Slice preparation}

For optogenetic activation experiments, PPR, excitability and spontaneous GABAergic event experiments: Mice were deeply anesthetized with ketamine-xylazine and perfused transcardially with ice-cold modified artificial cerebrospinal fluid (ACSF) bubbled with 95\% $\mathrm{O}_{2}-5 \% \mathrm{CO}_{2}$, and containing (in $\mathrm{mM}$ ): $2.5 \mathrm{KCl}, 26 \mathrm{NaHCO}_{3}, 1.25$ $\mathrm{Na}_{2} \mathrm{HPO}_{4}, 0.5 \mathrm{CaCl}_{2}, 10 \mathrm{MgSO}_{4}, 0.4$ ascorbic acid, 10 glucose, and 210 sucrose (optogenetic activation studies) or $3 \mathrm{KCl}, 26 \mathrm{NaHCO}_{3}, 1 \mathrm{NaH}_{2} \mathrm{PO}_{4}, 1 \mathrm{CaCl}_{2}, 1.5 \mathrm{MgCl}_{2}$, 
$124 \mathrm{NaCl}$ and 14 glucose (PPR, excitability and spontaneous GABAergic event measures). The brain was removed and sagittal or coronal slices (sectioned at a thickness of $275 \mu \mathrm{m}$ were obtained in ice-cold modified ACSF. Slices were then submerged in ACSF, bubbled with $95 \% \mathrm{O}_{2}-5 \% \mathrm{CO}_{2}$, and containing (in $\mathrm{mM}$ ): $2.5 \mathrm{KCl}$, $126 \mathrm{NaCl}, 26 \mathrm{NaHCO}_{3}, 1.25 \mathrm{Na}_{2} \mathrm{HPO}_{4}, 2 \mathrm{CaCl}_{2}, 2 \mathrm{MgSO}_{4}$, and 10 glucose and stored at room temperature for at least $1 \mathrm{~h}$ prior to recording (optogenetic activation studies), or 3 $\mathrm{KCl}, 26 \mathrm{NaHCO}_{3}, 1 \mathrm{NaH}_{2} \mathrm{PO}_{4}, 2 \mathrm{CaCl}_{2}, 1 \mathrm{MgCl}_{2}, 124 \mathrm{NaCl}$ and 14 glucose, incubated at $32^{\circ} \mathrm{C}$ for 45 then held at room temperature until recording (PPR, excitability and spontaneous GABAergic event measures).

For PV-FSI experiments: Mice were deeply anaesthetized with isoflurane and decapitated. The brain was removed and immersed in ice-cold cutting solution containing $205 \mathrm{mM}$ sucrose, $10 \mathrm{mM}$ glucose, $25 \mathrm{mM}$ NaHCO3, $2.5 \mathrm{mM} \mathrm{KCl,} 1.25 \mathrm{mM}$ $\mathrm{NaH} 2 \mathrm{PO} 4,0.5 \mathrm{mM} \mathrm{CaCl} 2$ and $7.5 \mathrm{mM} \mathrm{MgCl}$. Parasagittal brain slices (thickness 250 $\mathrm{mm}$ ) were prepared with a Leica VT $1000 \mathrm{~S}$ vibratome and incubated for 30 - $60 \mathrm{~min}$ at $34^{\circ} \mathrm{C}$ in a submerged chamber filled with artificial cerebrospinal fluid (ACSF) saturated with $95 \%$ oxygen and 5\% carbon dioxide. ACSF was composed of $125 \mathrm{mM} \mathrm{NaCl}, 25$ mM glucose, 25 mM NaHCO3, 2.5 mM KCl, 2 mM CaCl2, 1.25 mM NaH2PO4, 1 mM $\mathrm{MgCl}$. Subsequently, slices were kept for at least $30 \mathrm{~min}$ at room temperature before recording.

Slice visualization, electrophysiology and optogenetic stimulation

For optogenetic activation experiments: The slices were transferred to the recording chamber mounted on an Olympus BX51 upright, fixed-stage microscope and perfused with oxygenated ACSF at room temperature. A 60X, 0.9 NA water-immersion objective was used to examine the slice using Dodt contrast video microscopy. Patch pipette resistance was typically 3-4 $\mathrm{M} \Omega$ when filled with recording solutions. In voltageclamp experiments of IPSCs in SPNs the intracellular solution contained (in $\mathrm{mM}$ ): 127.5 $\mathrm{CsCH}_{3} \mathrm{SO}_{3}, 7.5 \mathrm{CsCl}, 10 \mathrm{HEPES}, 10 \mathrm{TEA}-\mathrm{Cl}, 4$ phosphocreatine disodium, 0.2 EGTA, $0.21 \mathrm{Na} 2 \mathrm{GTP}$, and $2 \mathrm{Mg}_{1.5} \mathrm{ATP}$ ( $\mathrm{pH}=7.3$ with $\mathrm{CsOH}, 280-290 \mathrm{mOsm} / \mathrm{kg}$ ). For whole-cell current clamp recordings from SPNs and cell-attached recordings from CINs the pipette contained (in mM): $135.5 \mathrm{KCH}_{3} \mathrm{SO}_{4}, 5 \mathrm{KCl}, 2.5 \mathrm{NaCl}, 5 \mathrm{Na}$-phosphocreatine, 10 
HEPES, 0.2 EGTA, 0.21 Na. GTP, and $2 \mathrm{Mg}_{1.5 A T P}(\mathrm{pH}=7.3$ with $\mathrm{KOH}, 280-290$ $\mathrm{mOsm} / \mathrm{kg}$ ). Electrophysiological recordings were obtained with a Multiclamp 700B amplifier (Molecular Devices, Sunnyvale, CA). Junction potential, which was 7-8 mV, was not corrected. Signals were digitized at $10 \mathrm{kHz}$ and logged onto a personal computer with the Winfluor software (John Dempster, University of Strathclyde, UK). Blue light LED (470 nm, Mightex, Toronto, Ontario, Canada) was used for full-field illumination via the objective. Single pulses were $1 \mathrm{~ms}$ long. In order to compare action potential (AP) latency and afterdepolarization (ADP) amplitude among SPNs the LED intensity was set to generate a just suprathreshold response in each SPN recorded.

For PPR, excitability and spontaneous GABAergic event experiments: The slices were transferred to the recording chamber mounted on a modified Ultima laser scanning microscope system (Bruker Nano) (excitability and spontaneous GABAergic event experiments) or an Olympus BX51W1 microscope (PPR experiments) and perfused with oxygenated ACSF at room temperature. A 60X, 1.0 NA Olympus LUMPFL waterimmersion objective was used to visualize slices, and fluorescently labeled neurons identified for recordings with the aid of a Dodt contrast image displayed in registration with the fluorescence image for experiments requiring identification of dSPNs vs iSPNs. Patch pipette resistance was typically 3-6 $\mathrm{M} \Omega$ when filled with recording solutions. For voltage-clamp recordings the intracellular solution contained (in $\mathrm{mM}$ ): $120 \mathrm{CsMeSO}_{3}, 5$ $\mathrm{NaCl}, 10$ TEA-Cl (tetraethylammonium-Cl), 10 HEPES, 5 Qx-314, 4 ATP-Mg ${ }^{2+}, 0.3$ GTP-Na+, 0.25 EGTA, and 0.05 Alexa Fluor 568 hydrazide $\mathrm{Na}^{+}$salt (Alexa Fluor was omitted in PPR experiments). For current clamp recordings the internal solution contained (in mM): $135 \mathrm{KMeSO}_{4}, 5 \mathrm{KCl}, 10 \mathrm{HEPES}, 2 \mathrm{ATP}-\mathrm{Mg}^{2+}$, $0.5 \mathrm{GTP}-\mathrm{Na}^{+}, 5$ phosphocreatine-tris, 5 phosphocreatine-Na+, 0.1 Fluo-4 pentapotassium salt, and 0.05 Alexa Fluor 568 hydrazide $\mathrm{Na}^{+}$salt. Patched SPNs were allowed to equilibrate for 10 min after rupture. Recordings were made using a Multiclamp 700B amplifier and either PrairieView 5.0 software (Bruker) or custom MATLAB protocols. sIPSCs were recorded in voltage-clamp mode at $+10 \mathrm{mV}$ for 180 seconds before and after bath application of drug. Access resistance and holding current were monitored throughout experiments and cells were excluded if these values changed by more than $20 \%$. Local electrical stimulation was evoked using a concentric bipolar stimulation electrode (FHC, Inc., 
Bowdoin, ME) placed near the cell using a stimulating amplitude of $\sim 1-3 \mathrm{mV}$ and $50 \mathrm{~ms}$ between stimuli pulses; 10 sweeps (10 s inter-sweep interval) were averaged per cell; cells were held at $-70 \mathrm{mV}$. PPR experiments were performed at $26-29^{\circ} \mathrm{C}$. Rheobase \& action potential firing frequencies were determined in current-clamp mode via a step series of $500 \mathrm{~ms}$ current injections starting at $-200 \mathrm{pA}$ and increasing by $25 \mathrm{pA}$ with each step. Resting membrane potential \& cell morphology were monitored throughout experiments and cells were excluded if resting at a more depolarized potential than $77.0 \mathrm{mV}$.

For PV-FSI experiments: Whole-cell patch clamp recordings were obtained in oxygenated ACSF at $35^{\circ} \mathrm{C}$. Neurons were visualized using infrared differential interference contrast (IR-DIC) microscopy (Zeiss FS Axioskop, Oberkochen, Germany). tdTomato expressing neurons were identified by switching to epifluorescence using a mercury lamp (X-cite, 120Q, Lumen Dynamics). Borosilicate pipettes of 5 - $8 \mathrm{MOhm}$ resistance were pulled with a Flaming / Brown micropipette puller P-1000 (Sutter instruments). All recordings were done in current clamp with an intracellular solution containing $130 \mathrm{mM} \mathrm{K}$-gluconate, $5 \mathrm{mM} \mathrm{KCl}, 10 \mathrm{mM}$ HEPES, $4 \mathrm{mM} \mathrm{Mg-ATP,} 0.3 \mathrm{mM}$ GTP, 10 mM Na2-phospho- creatine (pH 7.25, osmolarity 285 mOsm). Recordings were amplified using a MultiClamp 700B amplifier (Molecular Devices, CA, USA), filtered at 2 $\mathrm{kHz}$, digitized at 10-20 kHz using ITC-18 (HEKA Elektronik, Instrutech, NY, USA), and acquired using custom-made routines running on Igor Pro (Wavemetrics, OR, USA). Liquid junction potential was not corrected. Throughout all recordings pipette capacitance and access resistance were compensated for and data were discarded when access resistance increased beyond $30 \mathrm{MOhm}$. The intrinsic properties of the neurons were determined by a series of hyperpolarizing and depolarizing current steps and ramps, enabling the extraction of sub- and suprathreshold properties. All neurons were recorded in control conditions and after $>5 \mathrm{~min}$ of bath application of $1 \mu \mathrm{M} \mathrm{DH \beta E}$ (Tocris).

\section{Drugs and Reagents}

Experiments in Thy1-ChR2 mice were performed in the presence or absence of synaptic receptor blockers including $10 \mu \mathrm{M}$ SR-95531 (gabazine) to block GABAA 
receptors, $2 \mu \mathrm{M}$ CGP-55845 to block GABAв receptors, $10 \mu \mathrm{M}$ mecamylamine, a nonselective nAChR-antagonist and 1 or $10 \mu \mathrm{M}$ Dihydro- $\beta$-erythroidine hydrobromide $(\mathrm{DH} \beta E)$, a competitive $\alpha 4 \beta 2$ nAChR antagonist. Experiments were conducted with various combinations of the blockers. The acute effects of solution exchanges were measured at least 5 min after wash on. All drugs and reagents were acquired from Tocris (Ellisville, MO, USA) or HelloBio (Bristol, Avonmouth, UK).

\section{Data Analysis and Statistics}

Data analysis was performed using custom-made code in MATLAB (MathWorks, Natick, MA, USA) or Python; sIPSCs were analyzed using the Mini Analysis program (Synaptosoft). Two-tailed Wilcoxon signed-rank test (SRT) was used to test for changes of medians in matched-paired comparisons. The null hypotheses were rejected if the $P$ values were below 0.05. Boxplots represent range (whiskers), median (thick bar) and lower and upper quartiles. To analyze responses to stimulation, peristimulus time histograms (PSTH) were generated. PSTH were estimated using 20 ms wide bins. IV relationships, sIPSC frequencies and inter-event intervals were analyzed by two-way ANOVA.

Funding: BSF grant 2017020 to JLP and JAG. ISF grant 154/14 to JAG. ERC Consolidator (646880) to JAG. R01 NS104089/NINDS and NS022061/NINDS to JLP. Swedish Brain Fund (Hjärnfonden) grant to GS (FO2021-0333) and YJ (PS2020-0020). Swedish Research Council (VR-M) grant to GS (2019-01254). GS is supported by a Wallenberg Academy Fellowship (KAW 2017.0273).

\section{Figure Captions}

Fig. 1. nAChR-dependent inhibition and delay of spike latency in spiny projection neurons (SPNs) activated by corticostriatal fibers. A. Left: diagram of recording configuration. An SPN is patched in an acute slice from a ChAT-ChR2 mouse. Right: 1ms-long $470 \mathrm{~nm}$ LED pulse of increasing intensity generates excitatory postsynaptic potentials (EPSPs), or an AP followed by an after depolarization (ADP) in an SPN. B. Examples of the effect of $10 \mu \mathrm{M}$ mecamylamine (mec), an nAChR antagonist, on EPSP 
amplitude (dashed) or spike latency and ADP amplitude (solid). Mecamylamine significantly shortens spike latencies $\left(p=4.8 \cdot 10^{-4}, \mathrm{n}=12\right.$ SPNs, SRT) (C), as well as $\operatorname{ADP}\left(p=2.4 \cdot 10^{-3}, \mathrm{n}=12 \mathrm{SPNs}, \mathrm{SRT}\right)(\mathbf{D})$ and EPSP $(p=0.016, \mathrm{n}=7$ SPNs, SRT) (E) amplitudes. F. Examples of the effect of mecamylamine on latency of a spike triggered synaptically from a depolarized ("up-state") potential with an LED pulse. G. Mecamylamine significantly shortens "up-state" spike latencies $(p=0.03, \mathrm{n}=6$ SPNs, SRT) - two-sided Wilcoxon Signed-Rank test (SRT). ${ }^{* *} p<0.001,{ }^{* *} p<0.01,{ }^{*} p<$ 0.05 .

Fig. 2. Blockade of mecamylamine-insensitive $\alpha 7 \mathrm{nAChRs}$ with MLA increases SPN AP latency. A. Example responses of an SPN to paired pulse stimulation (evoked by two local electrical stimuli separated by $50 \mathrm{~ms}$ ), before and in the presence of mecamylamine $(10 \mu \mathrm{M})$. B. Mecamylamine had no effect on the EPSC amplitude evoked by the first paired stimulus (P1) ( $p=0.59, \mathrm{n}=15$ SPNs, SRT). C. Mecamylamine had no effect on the paired pulse ratio (P2/P1, $p=0.33, \mathrm{n}=15 \mathrm{SPNs}$, SRT). D. Example responses of MLA (2-5 $\mu \mathrm{M})$ on optogenetically-evoked AP latency. $\mathbf{E}$. MLA delayed AP latency in SPNs after full field optogenetic stimulation of corticostriatal inputs $(p=0.039, \mathrm{n}=9$, SRT $)$.

Fig. 3. The nAChR-dependent inhibition of corticostriatal SPN activation is mediated through and saturates GABAergic inhibition. A. Example of the occlusion of the mecamylamine effect on optogenetic synaptic activation of SPNs by receptor (GABAR) antagonists, $10 \mu \mathrm{M}$ SR-95531 (GABA ${ }_{A} R$ antagonist) and $2 \mu \mathrm{M}$ CGP-55845 (GABAB $R$ antagonist). Distribution of spike latencies (B) and ADP amplitude (C) in response to application of GABAR antagonists followed by mecamylamine, show that application of GABAR antagonist significantly shortens the AP latency $\left(p=4.6 \cdot 10^{-3}, \mathrm{n}=\right.$ 13 SPNs, SRT) and enhancement of the ADP amplitude $\left(p=2.4 \cdot 10^{-4}, \mathrm{n}=13 \mathrm{SPNs}\right.$, SRT). In contrast, the subsequent mecamylamine application fails to further shorten the AP latency ( $p=1 / 16, \mathrm{n}=5$ SPNs, SRT) or further enhance the ADP amplitude ( $p=1, \mathrm{n}=$ 5 SPNs, SRT). D. Example of how the mecamylamine effect saturates the GABAergic inhibition 
of the optogenetic synaptic activation of SPNs. E-F. Distribution of spike latencies (E) and ADP amplitude (F) in response to application of mecamylamine followed by GABAR antagonists, showed that the subsequent application of GABAR antagonists fails to further shorten the AP latency ( $p=1 / 4, n=5$ SPNs, SRT) or further enhance the ADP amplitude $(p=0.44, \mathrm{n}=5$ SPNs, SRT).

Fig. 4. The autonomous firing of cholinergic interneurons (CINs) enables them to respond more rapidly and vigorously than SPNs to feedforward corticostriatal excitation. A. Example of the autonomous discharge of a CIN recorded in cell-attached configuration (top) and the distribution of firing rates (bottom, $\mathrm{n}=15 \mathrm{CINs}$ ) in an acute striatal slice. B. Peristimulus histograms (PSTHs) of a CIN in response to optogenetic activation of corticostriatal fibers at various $470 \mathrm{~nm}$ LED intensities as compared to the amplitude of the EPSPs evoked in a nearby SPN (insets), demonstrates that CINs are much more sensitive than SPNs to feedforward cortical activation. C. Cumulative distribution of the latency to first spike of CINs recorded in cell-attached mode (green, $n$ = $8 \mathrm{CINs}$ ) as compared to the latency to first spike in SPNs recorded (from a restingstate) in whole-cell mode $(\mathrm{n}=12)$ before (black) and after (magenta) application of mecamylamine.

Fig. 5. Latency of feedforward GABAergic IPSCs in SPNs activated synaptically by corticostriatal fibers. A. Left: IPSCs recorded in an SPN held at $+10 \mathrm{mV}$ before (black) and after (red) application of GABAR antagonists, reveals GABA-sensitive (purple) IPSC. Right: Zoom in of the GABAergic IPSCs enables estimation (fit of diagonal black line) of the latency of the feed-forward GABA-sensitive inhibition. B. Distribution of the latency of feedforward GABAergic inhibition to SPNs in the Thy 1ChR2 mouse ( $n=7$ SPNs). C. Schematic demonstrating that the feedforward GABAergic inhibition precedes the earliest timing of feedforward cholinergic di-synaptic inhibition of SPNs, indicating that phasic activation of CINs cannot explain the nAChRdependent delay of spike latency. 
Fig 6. SPN intrinsic excitability is not increased by nAChR-blockade. A. Example IV traces of dSPNs and iSPNs in the absence and presence of $10 \mu \mathrm{M}$ mecamylamine. B. Left: resting membrane potential of dSPNs (black) and iSPNs (blue) in the absence and presence of mecamylamine (all SPNs: $p=0.43, \mathrm{n}=13$; dSPNs: $p=0.16, \mathrm{n}=7$; iSPNs: $p=0.87, \mathrm{n}=6$; SRT). Right: percent change in resting membrane potential after mecamylamine application. C. Left: rheobase current of dSPNs and iSPNs in the absence and presence of mecamylamine (all SPNs: $p=0.075, \mathrm{n}=13$; dSPNs: $p=0.5$, $\mathrm{n}=7$; iSPNs: $p=0.095, \mathrm{n}=6$; SRT). Right: percent change in rheobase current after mecamylamine application. D. Voltage responses to subthreshold current injections are not enhanced by mecamylamine ( $\mathrm{F} 11,120=0.86 p=0.58 ; \mathrm{n}=11$; two-way ANOVA), though a post-hoc Bonferroni test reveals a significant mecamylamine-induced decrease at $50 \mathrm{pA}(p<0.05)$ and $75 \mathrm{pA}(p<0.01)$ current injections. E. AP firing frequencies in response to suprathreshold somatic current injections were unaffected by mecamylamine $(\mathrm{F} 20,231=0.43, p=0.99 ; \mathrm{n}=12$; two-way ANOVA $)$.

\section{Fig 7. nAChR-blockade hyperpolarizes PV-FSI resting membrane potential and} reduces the frequency of sIPSCs in SPNs. A. Example recordings of sIPSCs from SPNs voltage-clamped at $+10 \mathrm{mV}$, before and during mecamylamine $(10 \mu \mathrm{M})$ application. B. Mecamylamine significantly enhanced sIPSC frequency in SPNs (F8,108 $=2.607, p=0.012 ; n=13$; two-way ANOVA). A post-hoc Bonferroni test revealed that this decrease was limited to low amplitude sIPSCs (20 pA bin: $p<0.001 ; 25$ pA bin: $p<$ 0.01). C. Mecamylamine caused a rightward shift in the cumulative probability distribution of sIPSC interevent intervals in SPNs (F20,252 $=2.10, p=4.7 \cdot 10^{-3} ; \mathrm{n}=13$; two-way ANOVA). D. Example traces of a PV-FSI before and after wash-in of $1 \mu \mathrm{M}$ $\mathrm{DH} \beta E$. E. Resting membrane potentials of PV-FSIs before and after wash-in of DH $\beta E$ (n $\left.=12 ; p=9.8 \cdot 10^{-4} ; \mathrm{SRT}\right)$. F. AP durations of PV-FSls in the absence and presence of $\mathrm{DH} \beta E(n=10 ; p=0.03 ; \mathrm{SRT})$. G. AP rise times of PV-FSIs in the absence and presence of DH $\beta E(n=10 ; p=0.02 ; S R T)$. 


\section{References}

Abudukeyoumu, N., T. Hernandez-Flores, M. Garcia-Munoz and G. W. Arbuthnott (2019).

"Cholinergic modulation of striatal microcircuits." Eur J Neurosci 49(5): 604-622.

Aceves Buendia, J. J., L. Tiroshi, W. H. Chiu and J. A. Goldberg (2019). "Selective remodeling of glutamatergic transmission to striatal cholinergic interneurons after dopamine depletion." Eur J Neurosci 49(6): 824-833.

Ade, K., Y. Wan, M. Chen, B. Gloss and N. Calakos (2011). "An Improved BAC Transgenic Fluorescent Reporter Line for Sensitive and Specific Identification of Striatonigral Medium Spiny Neurons." Frontiers in Systems Neuroscience 5(32).

Akins, P. T., D. J. Surmeier and S. T. Kitai (1990). "Muscarinic modulation of a transient K+ conductance in rat neostriatal neurons." Nature 344(6263): 240-242.

Albuquerque, E. X., E. F. Pereira, M. Alkondon and S. W. Rogers (2009). "Mammalian nicotinic acetylcholine receptors: from structure to function." Physiol Rev 89(1): 73-120.

Aosaki, T., M. Kimura and A. M. Graybiel (1995). "Temporal and spatial characteristics of tonically active neurons of the primate's striatum." J Neurophysiol 73(3): 1234-1252.

Aosaki, T., H. Tsubokawa, A. Ishida, K. Watanabe, A. M. Graybiel and M. Kimura (1994). "Responses of tonically active neurons in the primate's striatum undergo systematic changes during behavioral sensorimotor conditioning." J Neurosci 14(6): 3969-3984.

Apicella, P. (2017). "The role of the intrinsic cholinergic system of the striatum: What have we learned from TAN recordings in behaving animals?" Neuroscience 360: 81-94.

Apicella, P., E. Legallet and E. Trouche (1997). "Responses of tonically discharging neurons in the monkey striatum to primary rewards delivered during different behavioral states." Exp Brain Res 116(3): 456-466.

Arenkiel, B. R., J. Peca, I. G. Davison, C. Feliciano, K. Deisseroth, G. J. Augustine, M. D. Ehlers and G. Feng (2007). "In vivo light-induced activation of neural circuitry in transgenic mice expressing channelrhodopsin-2." Neuron 54(2): 205-218.

Assous, M. (2021). "Striatal cholinergic transmission. Focus on nicotinic receptors' influence in striatal circuits." Eur J Neurosci 53(8): 2421-2442.

Assous, M., J. Kaminer, F. Shah, A. Garg, T. Koós and J. M. Tepper (2017). "Differential processing of thalamic information via distinct striatal interneuron circuits." Nat Commun 8: 15860.

Assous, M. and J. M. Tepper (2019). "Excitatory extrinsic afferents to striatal interneurons and interactions with striatal microcircuitry." Eur J Neurosci 49(5): 593-603.

Beatty, J. A., M. A. Sullivan, H. Morikawa and C. J. Wilson (2012). "Complex autonomous firing patterns of striatal low-threshold spike interneurons." J Neurophysiol 108(3): 771-781.

Bennett, B. D. and C. J. Wilson (1999). "Spontaneous activity of neostriatal cholinergic interneurons in vitro." J Neurosci 19(13): 5586-5596. 
Bradfield, L. A., J. Bertran-Gonzalez, B. Chieng and B. W. Balleine (2013). "The thalamostriatal pathway and cholinergic control of goal-directed action: interlacing new with existing learning in the striatum." Neuron 79(1): 153-166.

Brimblecombe, K. R. and S. J. Cragg (2017). "The Striosome and Matrix Compartments of the Striatum: A Path through the Labyrinth from Neurochemistry toward Function." ACS Chem Neurosci 8(2): 235-242.

Cachope, R., Y. Mateo, B. N. Mathur, J. Irving, H. L. Wang, M. Morales, D. M. Lovinger and J. F. Cheer (2012). "Selective activation of cholinergic interneurons enhances accumbal phasic dopamine release: setting the tone for reward processing." Cell Rep 2(1): 33-41.

Calabresi, P., D. Centonze, P. Gubellini and G. Bernardi (1999). "Activation of M1-like muscarinic receptors is required for the induction of corticostriatal LTP." Neuropharmacology 38(2): 323-326.

Contant, C., D. Umbriaco, S. Garcia, K. C. Watkins and L. Descarries (1996). "Ultrastructural characterization of the acetylcholine innervation in adult rat neostriatum." Neuroscience 71(4): 937-947.

Crittenden, J. R., C. J. Lacey, F. J. Weng, C. E. Garrison, D. J. Gibson, Y. Lin and A. M. Graybiel (2017). "Striatal Cholinergic Interneurons Modulate Spike-Timing in Striosomes and Matrix by an Amphetamine-Sensitive Mechanism." Front Neuroanat 11: 20.

Dautan, D., I. Huerta-Ocampo, N. K. Gut, M. Valencia, K. Kondabolu, Y. Kim, T. V. Gerdjikov and J. Mena-Segovia (2020). "Cholinergic midbrain afferents modulate striatal circuits and shape encoding of action strategies." Nature Communications 11(1): 1739.

Day, M., D. Wokosin, J. L. Plotkin, X. Tian and D. J. Surmeier (2008). "Differential excitability and modulation of striatal medium spiny neuron dendrites." J Neurosci 28(45): 11603-11614.

DiFiglia, M. (1987). "Synaptic organization of cholinergic neurons in the monkey neostriatum." $\underline{J}$ Comp Neurol 255(2): 245-258.

Dorst, M. C., A. Tokarska, M. Zhou, K. Lee, S. Stagkourakis, C. Broberger, S. Masmanidis and G. Silberberg (2020). "Polysynaptic inhibition between striatal cholinergic interneurons shapes their network activity patterns in a dopamine-dependent manner." Nat Commun 11(1): 5113.

Ebihara, K., K. Yamamoto, K. Ueda, N. Koshikawa and M. Kobayashi (2013). "Cholinergic interneurons suppress action potential initiation of medium spiny neurons in rat nucleus accumbens shell." Neuroscience 236: 332-344.

Elghaba, R., N. Vautrelle and E. Bracci (2016). "Mutual Control of Cholinergic and LowThreshold Spike Interneurons in the Striatum." Frontiers in Cellular Neuroscience 10(111).

English, D. F., O. Ibanez-Sandoval, E. Stark, F. Tecuapetla, G. Buzsáki, K. Deisseroth, J. M. Tepper and T. Koos (2011). "GABAergic circuits mediate the reinforcement-related signals of striatal cholinergic interneurons." Nat Neurosci 15(1): 123-130.

Faust, T. W., M. Assous, F. Shah, J. M. Tepper and T. Koós (2015). "Novel fast adapting interneurons mediate cholinergic-induced fast GABAA inhibitory postsynaptic currents in striatal spiny neurons." Eur J Neurosci 42(2): 1764-1774. 
Faust, T. W., M. Assous, J. M. Tepper and T. Koós (2016). "Neostriatal GABAergic Interneurons Mediate Cholinergic Inhibition of Spiny Projection Neurons." J Neurosci 36(36): 9505-9511.

Gabel, L. A. and E. S. Nisenbaum (1999). "Muscarinic receptors differentially modulate the persistent potassium current in striatal spiny projection neurons." J Neurophysiol 81(3): 1418-1423.

Gerfen, C. R. and D. J. Surmeier (2011). "Modulation of striatal projection systems by dopamine." Annu Rev Neurosci 34: 441-466.

Gertler, T. S., C. S. Chan and D. J. Surmeier (2008). "Dichotomous Anatomical Properties of Adult Striatal Medium Spiny Neurons." The Journal of Neuroscience 28(43): 10814-10824.

Goldberg, J. A., J. B. Ding and D. J. Surmeier (2012). "Muscarinic modulation of striatal function and circuitry." Handb Exp Pharmacol(208): 223-241.

Goldberg, J. A. and J. N. Reynolds (2011). "Spontaneous firing and evoked pauses in the tonically active cholinergic interneurons of the striatum." Neuroscience 198: 27-43.

Graybiel, A. M. and C. W. Ragsdale, Jr. (1978). "Histochemically distinct compartments in the striatum of human, monkeys, and cat demonstrated by acetylthiocholinesterase staining." Proc Natl Acad Sci U S A 75(11): 5723-5726.

Hao, J. and T. G. Oertner (2012). "Depolarization gates spine calcium transients and spiketiming-dependent potentiation." Curr Opin Neurobiol 22(3): 509-515.

Ibáñez-Sandoval, O., H. S. Xenias, J. M. Tepper and T. Koós (2015). "Dopaminergic and cholinergic modulation of striatal tyrosine hydroxylase interneurons." Neuropharmacology 95: 468-476.

Johansson, Y. and G. Silberberg (2020). "The Functional Organization of Cortical and Thalamic Inputs onto Five Types of Striatal Neurons Is Determined by Source and Target Cell Identities." Cell Reports 30(4): 1178-1194.e1173.

Kimura, M., J. Rajkowski and E. Evarts (1984). "Tonically discharging putamen neurons exhibit set-dependent responses." Proc Natl Acad Sci U S A 81(15): 4998-5001.

Koós, T. and J. M. Tepper (1999). "Inhibitory control of neostriatal projection neurons by GABAergic interneurons." Nature Neuroscience 2(5): 467-472.

Koós, T. and J. M. Tepper (2002). "Dual cholinergic control of fast-spiking interneurons in the neostriatum." J Neurosci 22(2): 529-535.

Lapper, S. R. and J. P. Bolam (1992). "Input from the frontal cortex and the parafascicular nucleus to cholinergic interneurons in the dorsal striatum of the rat." Neuroscience 51(3): 533-545

Licheri, V., O. Lagström, A. Lotfi, M. H. Patton, H. Wigström, B. Mathur and L. Adermark (2018). "Complex Control of Striatal Neurotransmission by Nicotinic Acetylcholine Receptors via Excitatory Inputs onto Medium Spiny Neurons." J Neurosci 38(29): 6597-6607. 
Luo, R., M. J. Janssen, J. G. Partridge and S. Vicini (2013). "Direct and GABA-mediated indirect effects of nicotinic ACh receptor agonists on striatal neurones." The Journal of Physiology 591(1): 203-217.

Mesulam, M. M., D. Mash, L. Hersh, M. Bothwell and C. Geula (1992). "Cholinergic innervation of the human striatum, globus pallidus, subthalamic nucleus, substantia nigra, and red nucleus." J Comp Neurol 323(2): 252-268.

Morris, G., D. Arkadir, A. Nevet, E. Vaadia and H. Bergman (2004). "Coincident but distinct messages of midbrain dopamine and striatal tonically active neurons." Neuron 43(1): 133-143.

Muñoz-Manchado, A. B., C. Bengtsson Gonzales, A. Zeisel, H. Munguba, B. Bekkouche, N. G. Skene, P. Lönnerberg, J. Ryge, K. D. Harris, S. Linnarsson and J. Hjerling-Leffler (2018). "Diversity of Interneurons in the Dorsal Striatum Revealed by Single-Cell RNA Sequencing and PatchSeq." Cell Rep 24(8): 2179-2190.e2177.

Muñoz-Manchado, A. B., C. Foldi, S. Szydlowski, L. Sjulson, M. Farries, C. Wilson, G. Silberberg and J. Hjerling-Leffler (2014). "Novel Striatal GABAergic Interneuron Populations Labeled in the 5HT3aEGFP Mouse." Cerebral Cortex 26(1): 96-105.

Nelson, A. B., N. Hammack, C. F. Yang, N. M. Shah, R. P. Seal and A. C. Kreitzer (2014). "Striatal cholinergic interneurons Drive GABA release from dopamine terminals." Neuron 82(1): 63-70.

Pakhotin, P. and E. Bracci (2007). "Cholinergic Interneurons Control the Excitatory Input to the Striatum." The Journal of Neuroscience 27(2): 391-400.

Pawlak, V. and J. N. Kerr (2008). "Dopamine receptor activation is required for corticostriatal spike-timing-dependent plasticity." J Neurosci 28(10): 2435-2446.

Picciotto, Marina R., Michael J. Higley and Yann S. Mineur (2012). "Acetylcholine as a Neuromodulator: Cholinergic Signaling Shapes Nervous System Function and Behavior." Neuron 76(1): 116-129.

Planert, H., S. N. Szydlowski, J. J. J. Hjorth, S. Grillner and G. Silberberg (2010). "Dynamics of Synaptic Transmission between Fast-Spiking Interneurons and Striatal Projection Neurons of the Direct and Indirect Pathways." The Journal of Neuroscience 30(9): 3499-3507.

Plotkin, J. L. and J. A. Goldberg (2019). "Thinking Outside the Box (and Arrow): Current Themes in Striatal Dysfunction in Movement Disorders." Neuroscientist 25(4): 359-379.

Poppi, L. A., K. T. Ho-Nguyen, A. Shi, C. T. Daut and M. A. Tischfield (2021). "Recurrent Implication of Striatal Cholinergic Interneurons in a Range of Neurodevelopmental, Neurodegenerative, and Neuropsychiatric Disorders." Cells 10(4).

Prager, E. M. and J. L. Plotkin (2019). "Compartmental function and modulation of the striatum." J Neurosci Res 97(12): 1503-1514.

Raz, A., A. Feingold, V. Zelanskaya, E. Vaadia and H. Bergman (1996). "Neuronal synchronization of tonically active neurons in the striatum of normal and parkinsonian primates." J Neurophysiol 76(3): 2083-2088. 
Rice, M. E. and S. J. Cragg (2004). "Nicotine amplifies reward-related dopamine signals in striatum." Nat Neurosci 7(6): 583-584.

Shen, W., M. Flajolet, P. Greengard and D. J. Surmeier (2008). "Dichotomous dopaminergic control of striatal synaptic plasticity." Science 321(5890): 848-851.

Shuen, J. A., M. Chen, B. Gloss and N. Calakos (2008). "<em>Drd1a-</em>tdTomato BAC Transgenic Mice for Simultaneous Visualization of Medium Spiny Neurons in the Direct and Indirect Pathways of the Basal Ganglia." The Journal of Neuroscience 28(11): 2681-2685.

Silberberg, G. and J. P. Bolam (2015). "Local and afferent synaptic pathways in the striatal microcircuitry." Curr Opin Neurobiol 33: 182-187.

Solinas, M., M. Scherma, L. Fattore, J. Stroik, C. Wertheim, G. Tanda, W. Fratta and S. R. Goldberg (2007). "Nicotinic alpha 7 receptors as a new target for treatment of cannabis abuse." J Neurosci 27(21): 5615-5620.

Stern, E. A., D. Jaeger and C. J. Wilson (1998). "Membrane potential synchrony of simultaneously recorded striatal spiny neurons in vivo." Nature 394(6692): 475-478.

Tepper, J. M. and J. P. Bolam (2004). "Functional diversity and specificity of neostriatal interneurons." Curr Opin Neurobiol 14(6): 685-692.

Tepper, J. M., T. Koós, O. Ibanez-Sandoval, F. Tecuapetla, T. W. Faust and M. Assous (2018). "Heterogeneity and Diversity of Striatal GABAergic Interneurons: Update 2018." Frontiers in Neuroanatomy 12(91).

Tepper, J. M., T. Koós and C. J. Wilson (2004). "GABAergic microcircuits in the neostriatum." Trends Neurosci 27(11): 662-669.

Tepper, J. M., F. Tecuapetla, T. Koós and O. Ibáñez-Sandoval (2010). "Heterogeneity and diversity of striatal GABAergic interneurons." Front Neuroanat 4: 150.

Thomas, T. M., Y. Smith, A. I. Levey and S. M. Hersch (2000). "Cortical inputs to m2immunoreactive striatal interneurons in rat and monkey." Synapse 37(4): 252-261.

Threlfell, S., T. Lalic, N. J. Platt, K. A. Jennings, K. Deisseroth and S. J. Cragg (2012). "Striatal dopamine release is triggered by synchronized activity in cholinergic interneurons." Neuron 75(1): 58-64.

Wilson, C. J. and P. M. Groves (1981). "Spontaneous firing patterns of identified spiny neurons in the rat neostriatum." Brain Res 220(1): 67-80.

Wilson, C. J., Z. C. Xu, P. C. Emson and C. Feler (1990). "Anatomical and physiological properties of the cortical and thalamic innervations of neostriatal tissue grafts." Prog Brain Res 82: 417-426.

Zhou, F.-M., C. J. Wilson and J. A. Dani (2002). "Cholinergic interneuron characteristics and nicotinic properties in the striatum." Journal of Neurobiology 53(4): 590-605. 
bioRxiv preprint doi: https://doi.org/10.1101/2021.11.02 466870; this version posted December 6, 2021. The copyright holder for this preprint

(which was not certified by peer review) is the author/funder, who has granted bioRxiv a license to display the preprint in perpetuity. It is made available under aCC-BY-ND 4.0 International license.

Zhou, F. M., Y. Liang and J. A. Dani (2001). "Endogenous nicotinic cholinergic activity regulates dopamine release in the striatum." Nat Neurosci 4(12): 1224-1229.

Zucca, S., A. Zucca, T. Nakano, S. Aoki and J. Wickens (2018). "Pauses in cholinergic interneuron firing exert an inhibitory control on striatal output in vivo." Elife 7:e32510. 
A

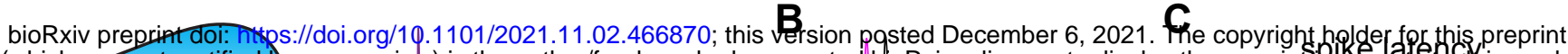
(which was not certified by peer review) is the author/funder, who has granted bloRxiv a license to display the preprinfinlfeerpaturtyclis is made
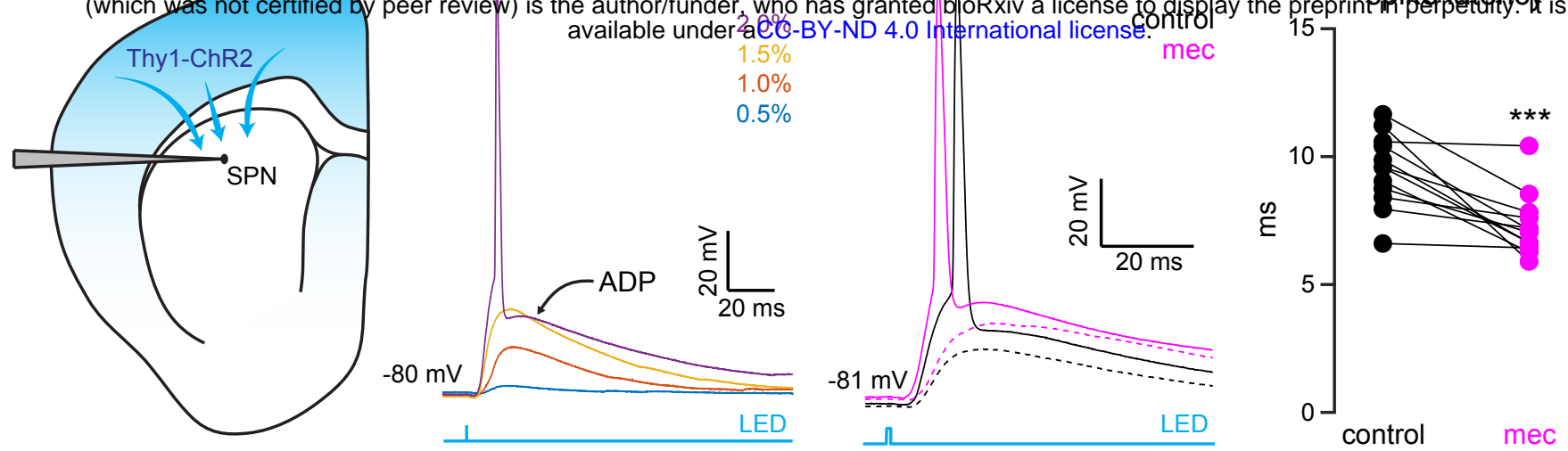

D

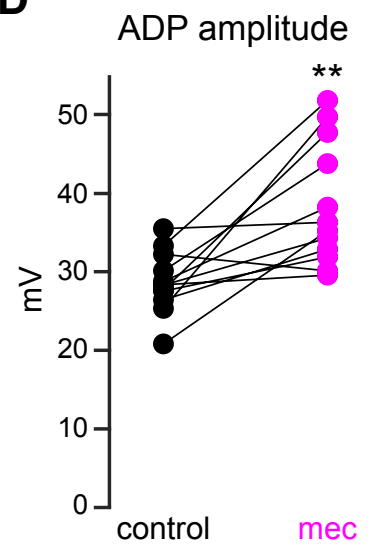

$\mathbf{E}$

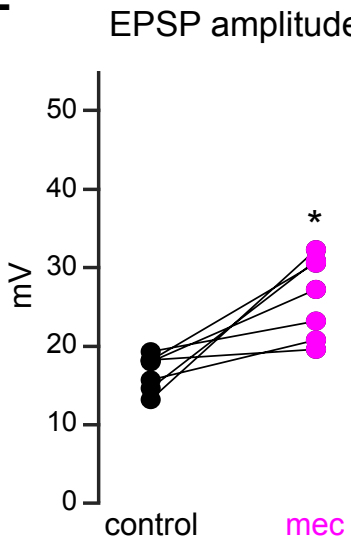

$\mathbf{F}$

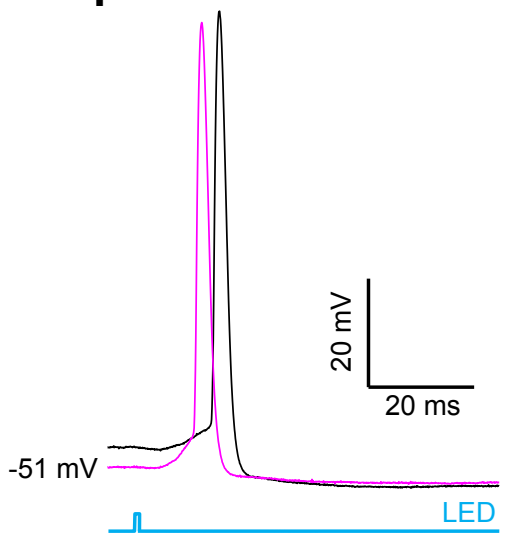

G

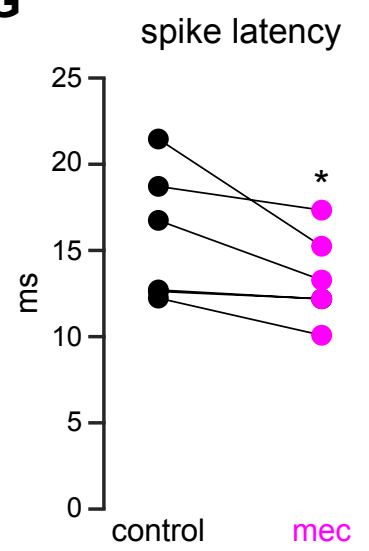

Fig. 1. nAChR-dependent inhibition and delay of spike latency in spiny projection neurons (SPNs) activated by corticostriatal fibers. A. Left: diagram of recording configuration. An SPN is patched in an acute slice from a ChAT-ChR2 mouse. Right: 1-ms-long $470 \mathrm{~nm}$ LED pulse of increasing intensity generates excitatory postsynaptic potentials (EPSPs), or an AP followed by an after depolarization (ADP) in an SPN. B. Examples of the effect of $10 \mu \mathrm{M}$ mecamylamine (mec), an nAChR antagonist, on EPSP amplitude (dashed) or spike latency and ADP amplitude (solid). Mecamylamine significantly shortens spike latencies $(p=4.8 \cdot 10-4, n=12$ SPNs, SRT) $(C)$, as well as ADP $(p=2.4 \cdot 10-3, n=12$ SPNs, SRT) $(D)$ and EPSP $(p=0.016, n=$ 7 SPNs, SRT) (E) amplitudes. F. Examples of the effect of mecamylamine on latency of a spike triggered synaptically from a depolarized ("up-state") potential with an LED pulse. G. Mecamylamine significantly shortens "up-state" spike latencies ( $p$ $=0.03, n=6$ SPNs, SRT) SRT - two-sided Wilcoxon Signed-Rank test (SRT). ${ }^{* *} p<0.001,{ }^{* *} p<0.01,{ }^{*} p<0.05$. 
A

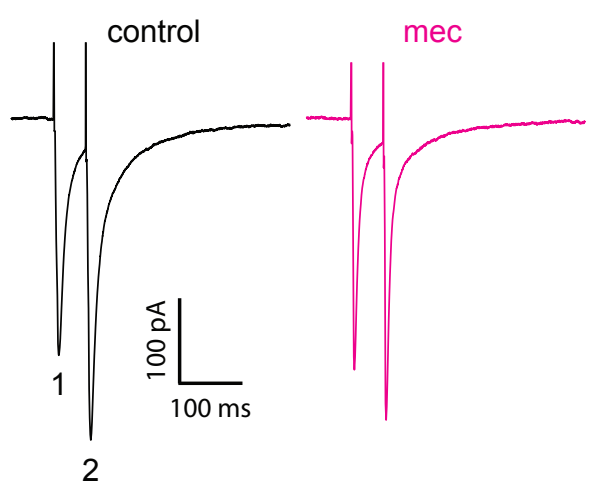

D

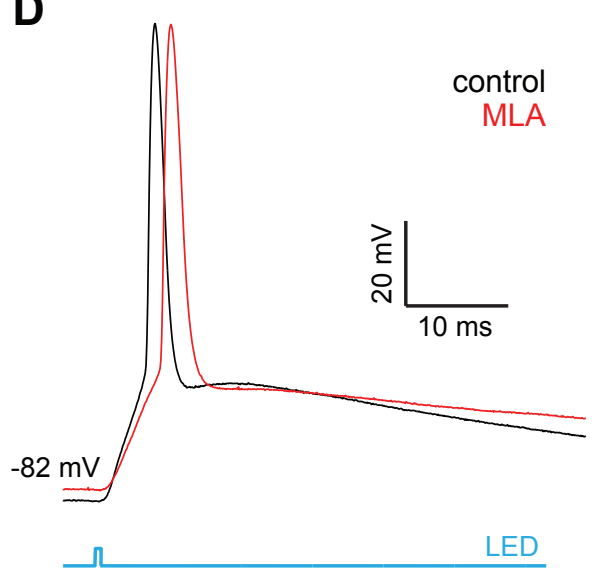

B

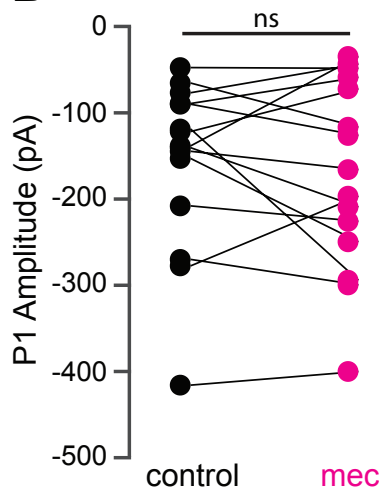

spike latency

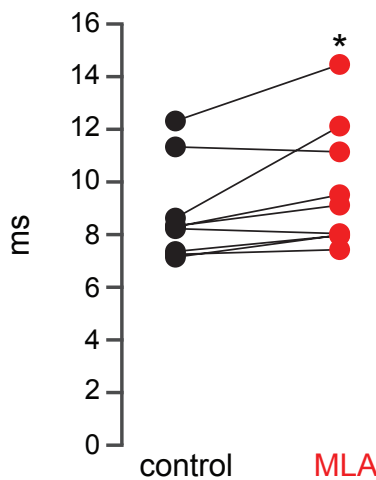

\section{E}

control MLA
C

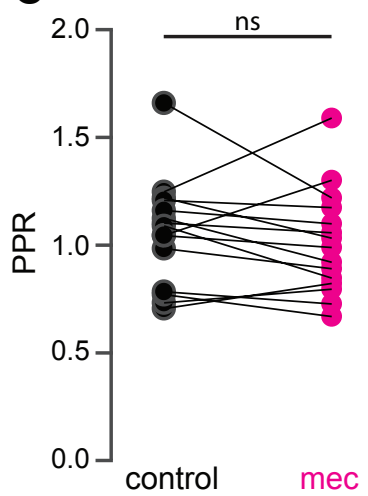

mec

Fig. 2. Blockade of mecamylamine-insensitive $\alpha 7 \mathrm{nAChRs}$ with MLA increases SPN AP latency. A. Example responses of an SPN to paired pulse stimulation (evoked by two local electrical stimuli separated by 50 ms), before and in the presence of mecamylamine $(10 \mu \mathrm{M})$. B. Mecamylamine had no effect on the EPSC amplitude evoked by the first paired stimulus $(P 1)$ ( $p=$ $0.59, n=15$ SPNs, SRT). C. Mecamylamine had no effect on the paired pulse ratio (P2/P1, $p=0.33, n=15$ SPNs, SRT). D. Example responses of MLA $(2-5 \mu \mathrm{M})$ on optogenetically-evoked AP latency. E. MLA delayed AP latency in SPNs after full field optogenetic stimulation of corticostriatal inputs $(p=0.039, n=9, S R T)$. 
bioRxiv preprint doi: https://doi.org/10.1101/2021.11.02.466870; this version posted December 6, 2021. The copyright holder for this preprint (which was not certified by peer review) is the author/funder, who has granted bioRxiv a license to display the preprint in perpetuity. It is made available under aCC-BY-ND 4.0 International license.

A
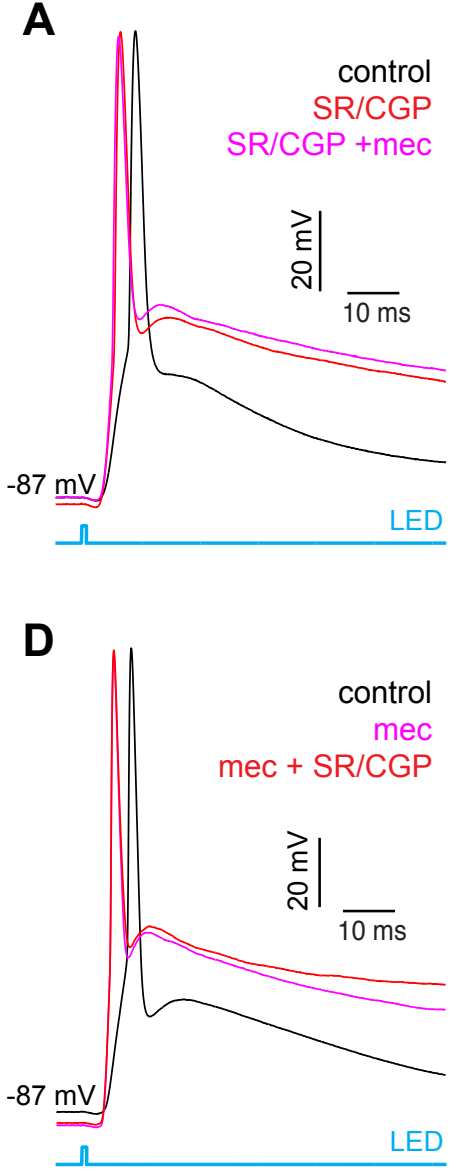

B

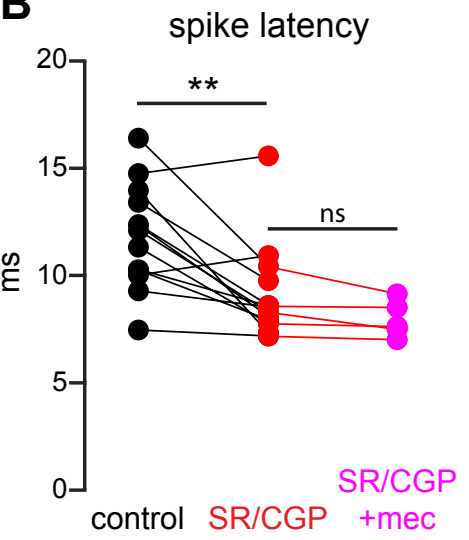

E

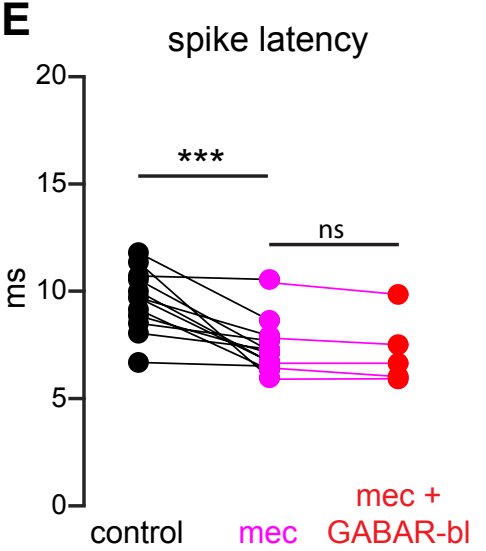

C

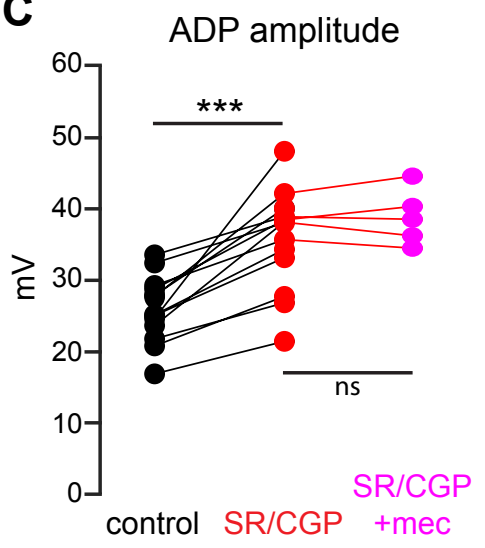

$\mathbf{F}$

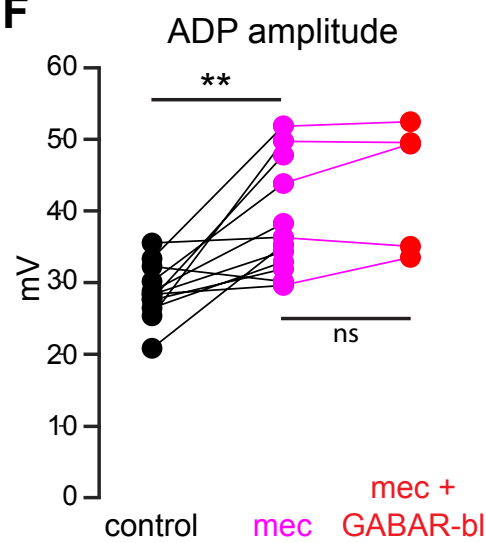

Fig. 3. The nAChR-dependent inhibition of corticostriatal SPN activation is mediated through and saturates GABAergic inhibition. A. Example of the occlusion of the mecamylamine effect on optogenetic synaptic activation of SPNs by receptor (GABAR) antagonists, $10 \mu \mathrm{M}$ SR-95531 (GABAAR antagonist) and $2 \mu \mathrm{M}$ CGP-55845 (GABABR antagonist). Distribution of spike latencies (B) and ADP amplitude (C) in response to application of GABAR antagonists followed by mecamylamine, show that application of GABAR antagonist significantly shortens the AP latency $(p=4.6 \cdot 10-3, n=13$ SPNs, SRT) and enhancement of the ADP amplitude $(p=2.4 \cdot 10-4, n=13$ SPNs, SRT). In contrast, the subsequent mecamylamine application fails to further shorten the AP latency ( $p=1 / 16, n=5$ SPNs, SRT) or further enhance the ADP amplitude $(p=1, n=5$ SPNs, SRT). D. Example of how the mecamylamine effect saturates the GABAergic inhibition of the optogenetic synaptic activation of SPNs. E-F. Distribution of spike latencies (E) and ADP amplitude (F) in response to application of mecamylamine followed by GABAR antagonists, showed that the subsequent application of GABAR antagonists fails to further shorten the AP latency $(p=1 / 4, n=5$ SPNs, SRT) or further enhance the ADP amplitude ( $p=0.44$, $\mathrm{n}=5$ SPNs, SRT). 
bioRxiv preprint doi: https://doi.org/10.1101/2021.111!02.466870; this version posted December 6, 2021. The copyright holder for this preprint (which was not certified by peer review) is the author/funder, who has granted bioRxiv a license to display the preprint in perpetuity. It is made
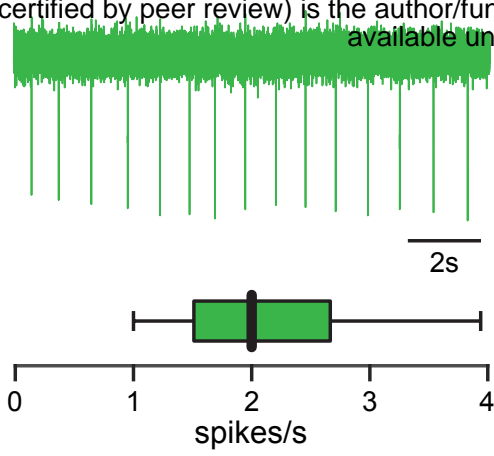

B
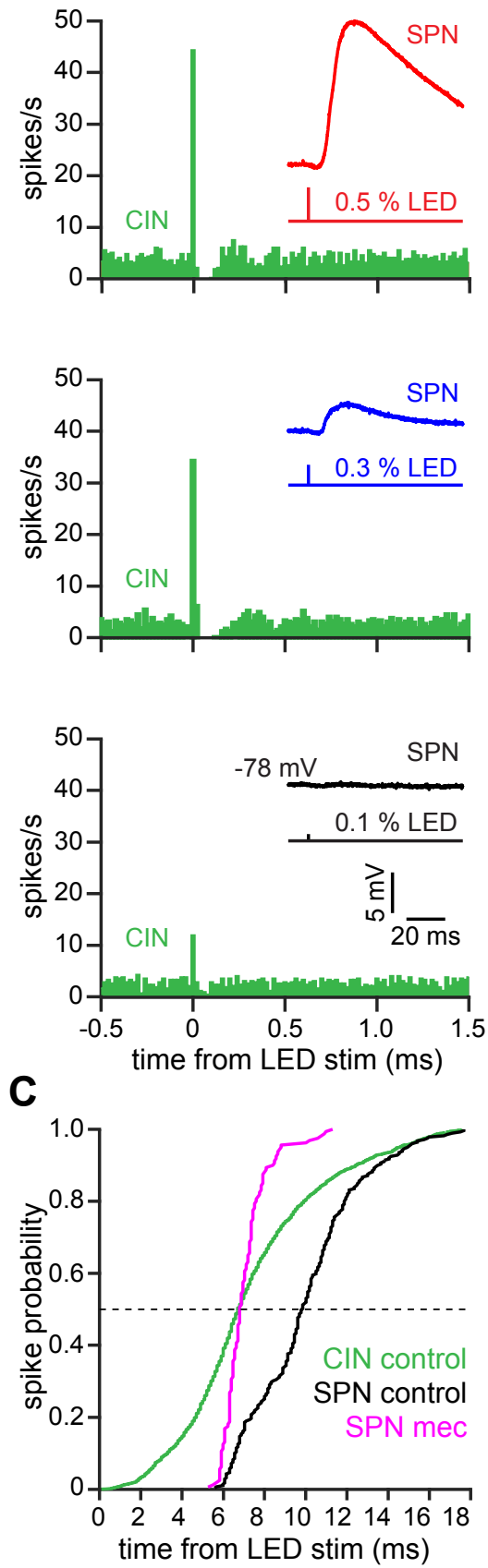

Fig. 4. The autonomous firing of cholinergic interneurons (CINs) enables them to respond more rapidly and vigorously than SPNs to feedforward corticostriatal excitation. A. Example of the autonomous discharge of a CIN recorded in cellattached configuration (top) and the distribution of firing rates (bottom, $n=15 \mathrm{CINs}$ ) in an acute striatal slice. B. Peristimulus histograms (PSTHs) of a CIN in response to optogenetic activation of corticostriatal fibers at various $470 \mathrm{~nm}$ LED intensities as compared to the amplitude of the EPSPs evoked in a nearby SPN (insets), demonstrates that CINs are much more sensitive than SPNs to feedforward cortical activation. C. Cumulative distribution of the latency to first spike of CINs recorded in cellattached mode (green, $n=8 \mathrm{CINs}$ ) as compared to the latency to first spike in SPNs recorded (from a resting-state) in whole-cell mode $(n=12)$ before (black) and after (magenta) application of mecamylamine. 
bioRxiv preprint doi: https://doi.org/10.1101/2021.11.02.466870; this version posted December 6, 2021. The copyright holder for this preprint (which was not certified by peer review) is the author/funder, who has granted bioRxiv a license to display the preprint in perpetuity. It is made available under aCC-BY-ND 4.0 International license.

A

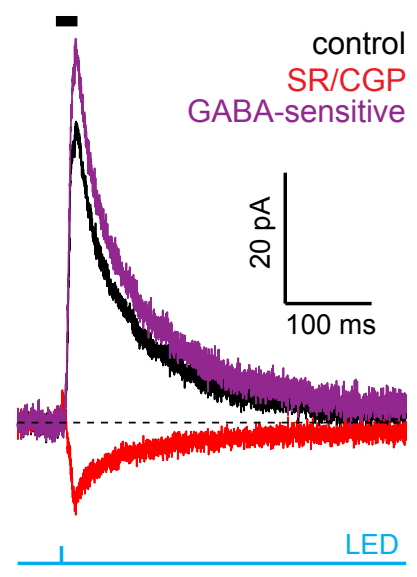

B

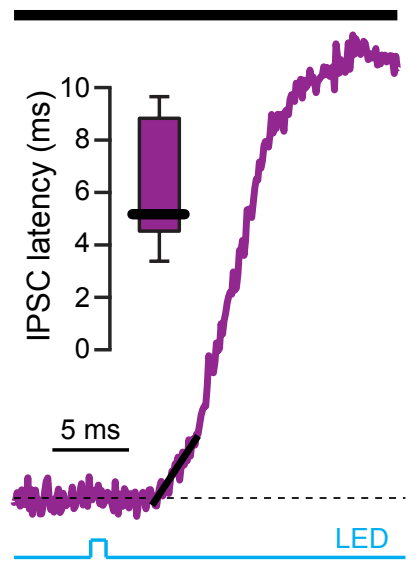

C

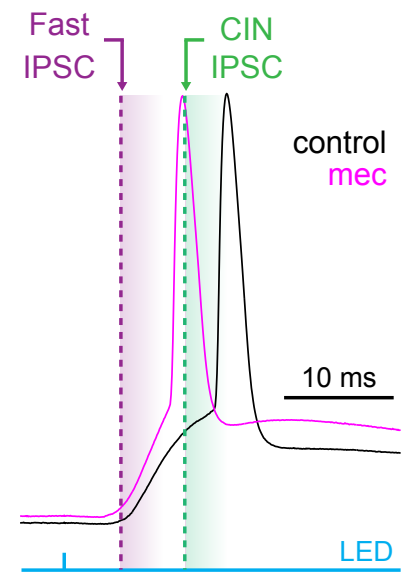

Fig. 5. Latency of feedforward GABAergic IPSCs in SPNs activated synaptically by corticostriatal fibers. A. Left: IPSCs recorded in an SPN held at $+10 \mathrm{mV}$ before (black) and after (red) application of GABAR antagonists, reveals GABA-sensitive (purple) IPSC. Right: Zoom in of the GABAergic IPSCs enables estimation (fit of diagonal black line) of the latency of the feed-forward GABAsensitive inhibition. B. Distribution of the latency of feedforward GABAergic inhibition to SPNs in the Thy1-ChR2 mouse ( $\mathrm{n}=7$ SPNs). C. Schematic demonstrating that the feedforward GABAergic inhibition precedes the earliest timing of feedforward cholinergic disynaptic inhibition of SPNs, indicating that phasic activation of CINs cannot explain the nAChR-dependent delay of spike latency. 
bioRxiv preprint doi: https://doi.org/10.1101/2021.11.02.466870; this version posted December 6, 2021. The copyright holder for this preprint

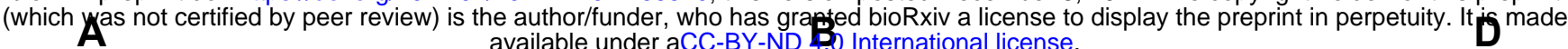

dSPN

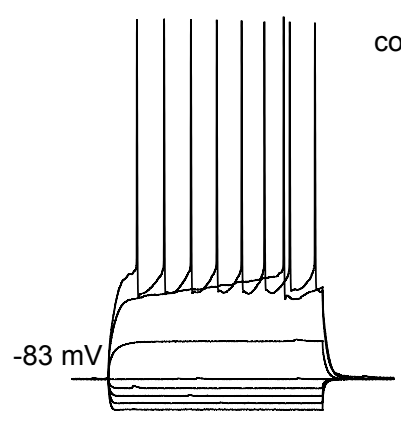

control
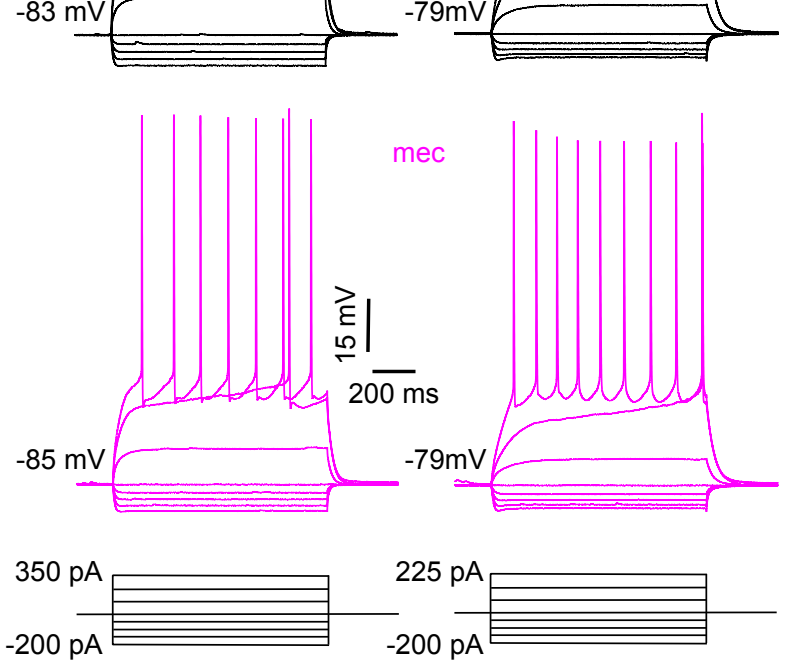

iSPN
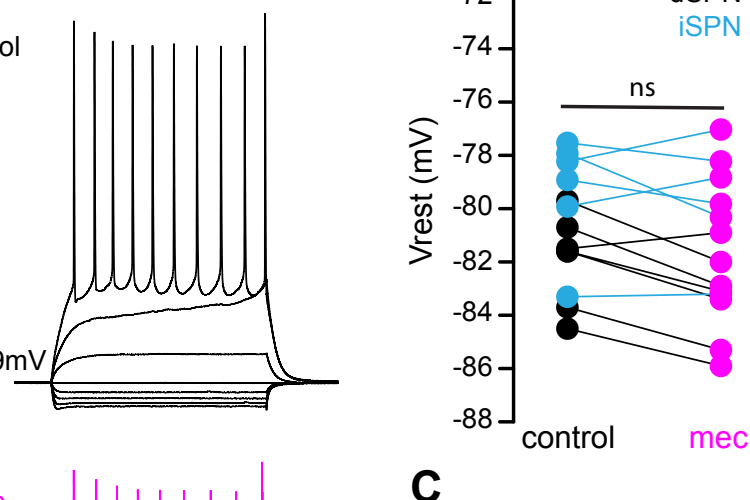

C

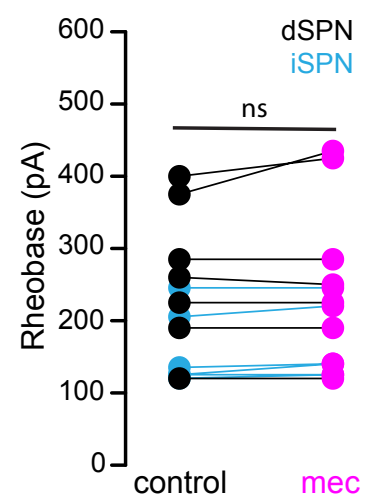

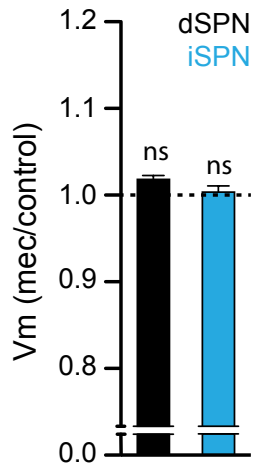

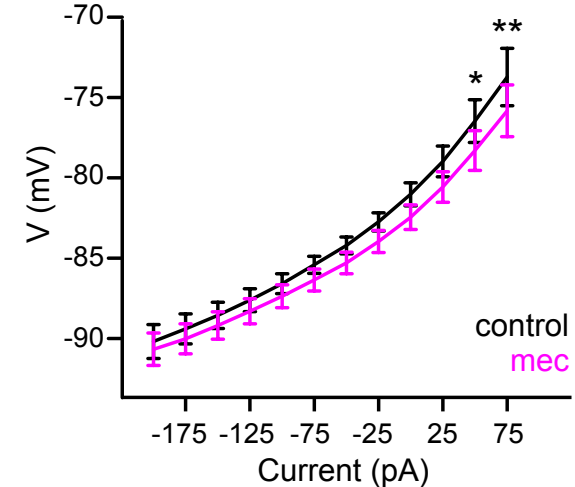

E
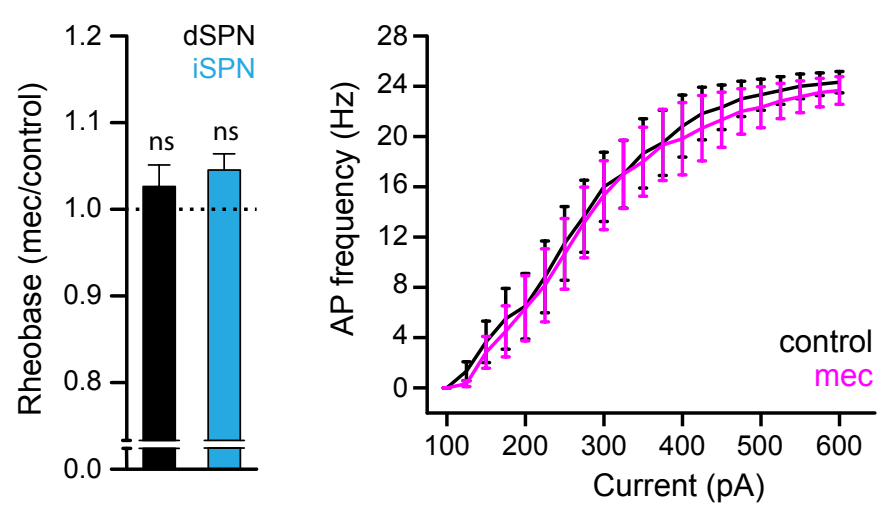

Fig 6. SPN intrinsic excitability is not increased by $n$ AChR-blockade. A. Example IV traces of dSPNs and iSPNs in the absence and presence of $10 \mu \mathrm{M}$ mecamylamine. B. Left: resting membrane potential of dSPNs (black) and iSPNs (blue) in the absence and presence of mecamylamine (all SPNs: $p=0.43, n=13$; dSPNs: $p=0.16, n=7$; iSPNs: $p=0.87, n=6$; SRT). Right: percent change in resting membrane potential after mecamylamine application. C. Left: rheobase current of dSPNs and iSPNs in the absence and presence of mecamylamine (all SPNs: $p=0.075, n=13$; dSPNs: $p=0.5, n=7 ;$ iSPNs: $p=0.095$, $n=6$; SRT). Right: percent change in rheobase current after mecamylamine application. D. Voltage responses to subthreshold current injections are not enhanced by mecamylamine $(F 11,120=$ $0.86 \mathrm{p}=0.58 ; \mathrm{n}=11$; two-way ANOVA), though a post-hoc Bonferroni test reveals a significant mecamylamine-induced decrease at $50 \mathrm{pA}(\mathrm{p}<0.05)$ and $75 \mathrm{pA}(\mathrm{p}<$ $0.01)$ current injections. E. AP firing frequencies in response to suprathreshold somatic current injections were unaffected by mecamylamine $(F 20,231=0.43, p=0.99$; $\mathrm{n}=12$; two-way ANOVA). 
bioRxiv preprint doi: https://doi.org/10.1101/2021.11.02.466870; this version posted December 6, 2021. The copyright holder for this preprint (which was not certified by peer review) is the author/funder, who has granted bioRxiv a license to display the preprint in perpetuity. It is made available under aCC-BY-ND 4.0 International license.

A

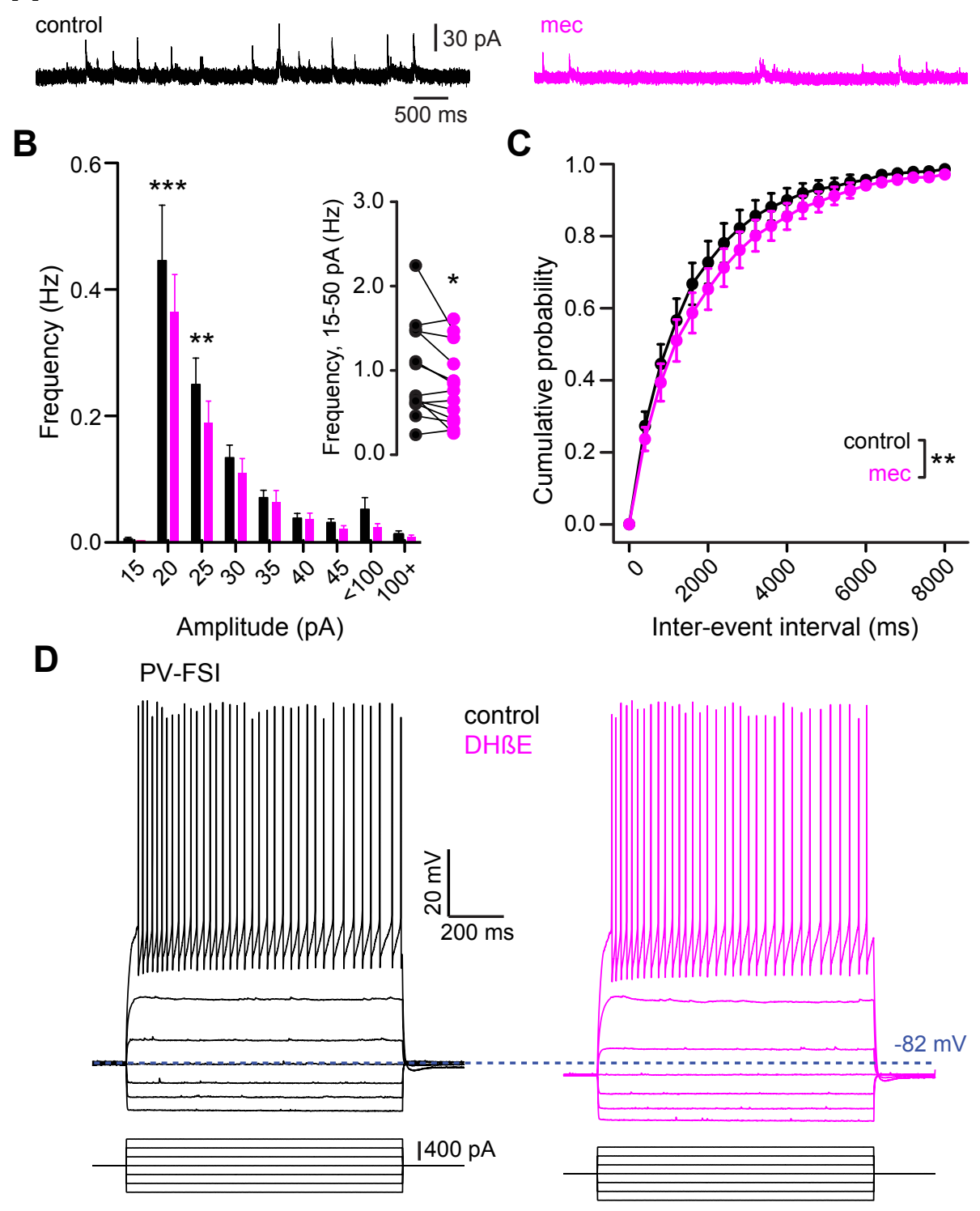

$\mathbf{E}$

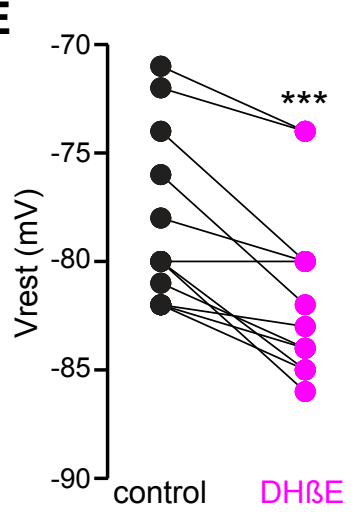

$\mathbf{F}$

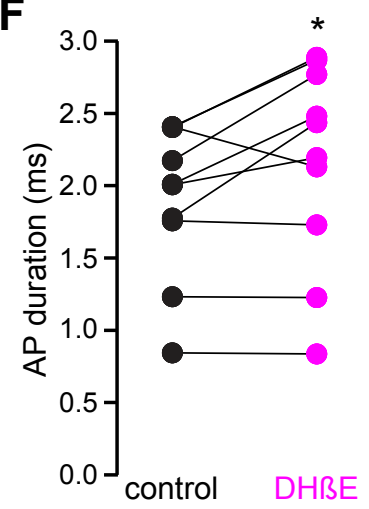

G

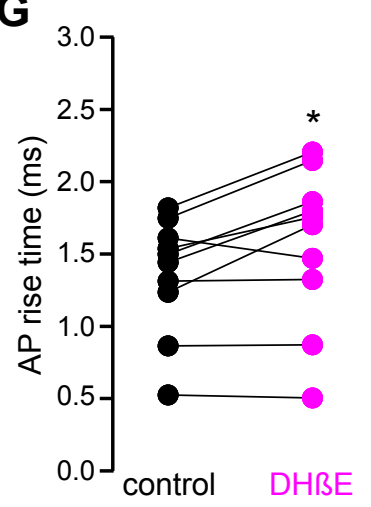

Fig 7. nAChR-blockade hyperpolarizes PV-FSI resting membrane potential and reduces the frequency of sIPSCs in SPNs. A. Example recordings of sIPSCs from SPNs voltage-clamped at $+10 \mathrm{mV}$, before and during mecamylamine $(10 \mu \mathrm{M})$ application. $\mathbf{B}$. Mecamylamine significantly enhanced sIPSC frequency in SPNs (F8,108 = 2.607, $p=0.012 ; n=13$; two-way ANOVA). A post-hoc Bonferroni test revealed that this decrease was limited to low amplitude sIPSCs (20 pA bin: $p<0.001 ; 25$ pA bin: $p<0.01$ ). C. Mecamylamine caused a rightward shift in the cumulative probability distribution of sIPSC interevent intervals in SPNs (F20,252 = 2.10, $p=4.7 \cdot 10-3 ; n=13$; two-way ANOVA). D. Example traces of a PV-FSI before and after wash-in of $1 \mu M$ DH $\beta E$. E. Resting membrane potentials of PV-FSIs before and after wash-in of DH $\beta E(n=12 ; p=9.8 \cdot 10-4$; SRT). F. AP durations of PV-FSIs in the absence and presence of $\mathrm{DH} \beta E(n=10 ; p=0.03 ;$ SRT). G. AP rise times of PV-FSIs in the absence and presence of $D H \beta E(n=10$; 